\title{
Classical and quantum integrable sigma models. Ricci flow, "nice duality" and perturbed rational conformal field theories
}

\author{
Vladimir Fateev \\ Université Montpellier 2, Laboratoire Charles Coulomb, \\ UMR 5221, F-34095, Montpellier, France \\ Landau Institute for Theoretical Physics, 142432 Chernogolovka, Russia
}

\begin{abstract}
We consider classical and quantum integrable sigma models and their relations with the solutions of renormalization group equations. We say that an integrable sigma model possesses the "nice" duality property if the dual quantum field theory has the weak coupling region. As an example, we consider the deformed $C P(n-1)$ sigma model with additional quantum degrees of freedom. We formulate the dual integrable field theory and use perturbed conformal field theory, perturbation theory, $S$-matrix, Bethe Ansatz and renormalization group methods to show that this field theory has the "nice" duality property. We consider also an alternative approach to the analysis of sigma models on the deformed symmetric spaces, based on the perturbed rational conformal field theories.
\end{abstract}




\section{Contents}

1 Introduction 2

2 Conformal field theory and reflection amplitudes

3 Deformed $C P(n-1)$ models with fermion and dual QFT 7

4 Action of dual QFT admitting perturbative expansion, non-local integrals and scattering theory

5 Bethe Ansatz analysis $\quad 11$

6 Sigma models, Ricci flow, integrability and observables 15

7 Conformal limit of metric, semiclassical reflection amplitudes and UV asymptotic of central charge

8 Integrable perturbation $\mu_{1} e^{b\left(e_{0} \cdot \varphi\right)} \quad 2$

9 Rational CFTs, their perturbations and sigma models $\quad 26$

10 Concluding Remarks 31

A Explicit expressions for the currents and densities of local integrals 33

B Possible parametrization of SU $(n) \quad 34$

\section{Introduction}

Duality plays an important role in the analysis of statistical, quantum field and string theory systems. Usually it maps a weak coupling region of one theory to the strong coupling region of the other and makes it possible to use perturbative, semiclassical and renormalization group methods in different regions of the coupling constant. For example, the well known duality between Sine-Gordon and massive Thirring models [1, 2] together with integrability plays an important role for the justification of exact scattering matrix [3] in these theories. Another well known example of the duality in two dimensional integrable systems is the weak-strong coupling flow from affine Toda theories to the same theories with dual affine Lie algebra [4], [5], 6]. The phenomenon of electric-magnetic duality in four dimensional $N=4$ supersymmetric gauge theories conjectured in [7, [8] and developed for $N=2$ theories in [9] (and in many subsequent papers) opens the possibility for the non-perturbative analysis of the spectrum and phase structure in supersymmetric gauge field theories. The remarkable field/string duality [11, [10] leads to the unification of the ideas and methods for the analysis of these seemingly different quantum systems.

While known for many years the phenomenon of duality in quantum field theory still looks rather mysterious and needs to be further analyzed. Such analysis crucially simplifies for two-dimensional integrable relativistic systems. These theories besides the Lagrangian formulation possess also an unambiguous definition in terms of factorized scattering theory, 
which contains all information about off- shell data of quantum theory. These data allow the use of non-perturbative methods for the calculation of observables in integrable field theories. The comparison of the observables calculated from the scattering data and from the perturbative, semiclassical or renormalization group analysis based on the Lagrangian formulation makes it possible in some cases to justify the existence of two different (dual) Lagrangian representations of a quantum theory.

The two particle factorized scattering matrix is a rather rigid object. It is constrained by the global symmetries, factorization equation and unitarity and crossing symmetry relations. After solving of these equations the scattering matrix $S$ can contain one (or more) free parameter. At some value of this parameter $\lambda=\lambda_{0}$ the scattering matrix $S\left(\lambda_{0}\right)$ becomes the identity matrix and has a regular expansion around this point. In many cases this expansion can be associated with the perturbative expansion of some Lagrangian theory with parameter $b$ near some free point. Sometimes there is a second point $\lambda=\lambda_{1}$ where $S(\lambda)$ reduces to identity matrix and admits a regular expansion in $\left(\lambda-\lambda_{1}\right)$. If this expansion can be associated with the perturbative expansion of another local Lagrangian at small coupling $\gamma=\gamma(b)$, then the two different Lagrangians describe the same theory, which has two different (dual) perturbative regimes.

A more interesting situation occurs when $S(\lambda)$ has a regular expansion in $\left(\lambda-\lambda_{0}\right)$ which agrees with perturbative expansion in $b$ of some field theory with local action $\mathcal{A}(b)$, but at the point $\lambda_{1}$ the $S$-matrix tends to some "rational" scattering matrix corresponding to the $S$-matrix of a non-linear sigma model on a symmetric space. Near the point $\lambda_{1}$ it can be considered as a deformation of a symmetric scattering. In this case it is natural to search for the dual theory as sigma model with target space looking as a deformed symmetric space. The metric and other characteristics of sigma model on the manifold is subject to very rigid conditions, namely non-linear renormalization group $(\mathrm{RG})$ equations [12]. If one has found the solution of RG equations which gives the observables in the sigma model theory, coinciding with those derived from the factorized $S$ - matrix theory one can conclude that field theory with the action $\mathcal{A}(b)$ is dual to a sigma model on the deformed symmetric space. The short distance behaviour of such theory can be studied by RG and conformal field theory (CFT) methods. The agreement of the CFT data, derived from the action $\mathcal{A}(b)$ (considered as a perturbed CFT) with the data derived from RG for sigma model gives an additional important test for the duality ("nice" duality).

The analysis of integrable quantum SMs on the deformed symmetric spaces and their dualities started in the papers [17,,[18, [16]. Later in the papers [19],20] the general classical SMs on the deformed groups and cosets manifolds have been constructed.. Unfortunately, not all these SMs, integrable classically, are integrable in quantum case. In particular it happens for the cosets having $U(1)$ group in denominator (see for example [34]). In many cases this situation can be improved by introduction of additional quantum degrees of freedom, which are invisible in the classical limit.

We say that an integrable SM has the "nice" duality if the dual integrable QFT has the weak coupling region. This implies that we can study this theory by different methods (perturbation theory, RG and CFT analysis) in different regimes. Note that the SMs with the property of "nice" duality form a very small subspace in the space of all integrable quantum SMs on the deformed symmetric spaces and such SMs with additional quantum degrees freedom.

A simple (but rather non-trivial) example is provided by the $C P(n-1)$ SM. This model is integrable classically but non-integrable (for $n>2$ ) at the quantum level. After adding 
the massless fermion interacting with $U(1)$ gauge field on $C P(n-1)$ (massless axion, linearly coupled with the density of topological charge ) this SM becomes integrable and its deformed version has the "nice" duality property. We study this theory in the main part of this paper. Here we say a few words about non-integrable $C P(n-1)$ SMs.

These models were intensively studied during 70-80s due to their's similarity with fourdimensional $S U(n)$ gauge QFTs. Namely, the $C P(n-1)$ SMs and $S U(n)$ gauge theories are asymptotically free, possess instantons, and manifest the phenomenon of confinement 1 It is natural that $C P(n-1)$ SMs served as baby-laboratory for the analysis of $S U(n)$ gauge theory, in particular, for analysis of instanton contributions [13] and lattice simulations [14].

The spectrum of the $C P(n-1) \mathrm{SMs}$ in $\frac{1}{n}$ approach was studied in [15]. It was shown that besides the basic particles, which are the only particles in the integrable version of this model, one has also the particles which are their bound states, confined by Coulomb forces. The addition of fermion (axion) produce an essential restructure of the spectrum. Of course, the influence of the axion on the spectrum of gauge theory is a more interesting and much more complicated problem.

The spectrum of the deformed non-integrable $C P(n-1)$ SMs seems to be qualitatively the same as that of the undeformed models and can not be studied by perturbative methods. Due to the "nice" duality, in the integrable $C P(n-1)$ SMs with axion there is a weak coupling region. In this region the basic particles also form the bound state, which disappear from the spectrum outside the perturbative region. It is possible however that in a non-integrable QFT they survive in the strong coupling (SM) regime. We hope to return to this problem in a future publication.

This paper is organized as follows. In section 2 we describe the basic CFTs, which can be formulated in terms of $2 n-1$ bosonic fields, and their primary fields are the exponents of these fields. We calculate the reflection amplitudes in these CFTs which are important for the calculation of UV asymptotics in perturbed CFTs. These amplitudes serve also for identification of CFTs in different representations. In particular, for justification of dual SM representations.

In section 3 we explain the general properties of deformed $C P(n-1)$ SMs with fermion and write the action of perturbed CFTs, constructed in section 2. We conjecture that these QFTs provide a dual description of deformed $C P(n-1)$ SMs with fermion.

In section 4 we represent the action of dual QFT in the form suitable for the perturbation theory in parameter $b$. We provide non-local integrals of motion which form the Borel subalgebra of $S U(n)_{\mathrm{q}}$ and generate $S U(n)_{\mathrm{q}}$ symmetry of the scattering theory. We describe the spectrum and scattering theory of this QFT.

In section 5 we use the Bethe Ansatz approach to derive the exact relations between the parameters of action and scattering theory in this QFT. We calculate the observables, which can be compared with the observables calculated using the dual SM description of our QFT.

In section 6 we consider classical and quantum integrable SMs on the deformed symmetric spaces. We discuss Ricci flows in these SMs and the relation between the parameters of integrable SMs with the parameters of their scattering theory. We calculate the observables in integrable deformed $C P(n-1) \mathrm{SM}$ and show that in the scaling (one loop) approximation they coincide with the observables calculated in the Bethe Ansatz approach.

In section 7 we consider the conformal limit of the deformed $C P(n-1)$ SMs. For simplicity

\footnotetext{
${ }^{1}$ For $n>2$, both theories have non-topological classical solutions, the role of which is not clear at the moment.
} 
of equations we consider the case $n=3$. We calculate the reflection amplitudes associated with this conformal SM and show that they coincide with reflection amplitudes calculated in section 2 i.e. these CFTs are dual. We use these amplitudes to get the UV asymptotics of effective central charge in the deformed $C P(n-1)$ SMs on the circle of length $R$.

In section 8 we study the second integrable perturbation of CFTs described in section 2. We study the scattering theory of these QFTs and see that at small and large values of the coupling constant $b$ they can be studied by perturbation theory in $b$ and $\frac{1}{b}$ i.e. it has two different dual representation. In the strong coupling regime they can be described by the action with the SM part coinciding with the conformal limit of the deformed $C P(n-1)$ SMs and the potential part described by tachyon. After a simple analytical continuation in the coupling constant the SM part of the actions become singular. These action describe integrable classical models but after the quantization these QFTs are well defined only for discrete values of coupling constant.

In section 9 we discuss the rational CFTs, which are closely related with conformal SMs with singular actions and the discrete values of coupling constant. We show that these CFTs perturbed by proper fields describe non-integrable deformed $C P(n-1)$ SMs with topological parameter and integrable deformed $C P(n-1)$ with fermion (axion). We consider more general

CFTs represented by the cosets $\frac{G_{m} \times G_{l}}{G_{m+l}}$ and study their deformations by different fields in different regions of integers $m, l$ and $h$ (Coxeter number of $G$ ). We study their RG propertied and show that these QFTs provide an independent description of a large variety of SMs on the deformed symmetric spaces. Finally we give a simple inequality which provides a necessary condition for a sigma model to have "nice" duality.

\section{Conformal field theory and reflection amplitudes}

We consider CFT, which has the hidden $s l(n)$ and $s l(n-1)$ - symmetries and can be described by $2 n-1$ fields

$$
\left\{\varphi, \vartheta, \vartheta_{0}, \vartheta_{n}\right\}=\left\{\left(\varphi_{1}, . ., \varphi_{n-1}\right),\left(\vartheta_{1}, . ., \vartheta_{n-2}\right), \vartheta_{0}, \vartheta_{n}\right\}
$$

We introduce the parameters $a, b$ satisfying the relation

$$
a^{2}-b^{2}=1
$$

and denote as $c$ the parameter

$$
c=\frac{\sqrt{n+b^{2}}}{\sqrt{n(n-1}}
$$

Let $h_{j}$ are $n$ vectors in $n-1$ dimensional space (the wights of fundamental representation of $s l(n))$ with the scalar products:

$$
h_{i} \cdot h_{j}=\delta_{i, j}-\frac{1}{n}
$$

and $\eta_{j}$ the vectors in $n-2$ dimensional space (the wights of fundamental representation of $\left.\operatorname{sl}(n-1), \eta_{i} \cdot \eta_{j}=\delta_{i, j}-\frac{1}{n-1}\right)$. Then $2(n-1)$ "screening charges" can be written in the form of $(n-1)$ pairs of fields $\left\{V_{1}, V_{-2}\right\},\left\{V_{2}, V_{-3}\right\}, . .,\left\{V_{n-1}, V_{-n}\right\}$, where $\left\{V_{k}, V_{-k-1}\right\}(k=1, . ., n-1)$ is:

$$
\left\{\mu \exp \left(b\left(h_{k} \cdot \varphi\right)+\mathrm{i} a\left(\eta_{k} \cdot \vartheta\right)+\mathrm{i} c \vartheta_{0}\right), \mu \exp \left(-b\left(h_{k+1} \cdot \varphi\right)-\mathrm{i} a\left(\eta_{k} \cdot \vartheta\right)-\mathrm{i} c \vartheta_{0}\right)\right\}
$$

We introduce $n-1$ and $n-2$ dimensional vectors $\rho$ and $\rho_{1}$, which are the halves of sum of the roots of $\operatorname{sl}(n)$, and $s l(n-1)$ and the dilaton field

$$
\Phi=\frac{1}{b}(\rho \cdot \varphi)+\frac{\mathrm{i}}{a}\left(\rho_{1} \cdot \vartheta\right)=(Q \cdot \varphi)+\mathrm{i}\left(Q_{1} \cdot \vartheta\right) .
$$


Then the theory described by the action $\mathcal{A}_{C F T}=\mathcal{A}_{0}+\mathcal{A}_{C}$, where $\mathcal{A}_{0}=\int d^{2} x \frac{1}{8 \pi}\left(\partial_{\mu} \vartheta_{n} \partial_{\mu} \vartheta_{n}\right)$ is the action of a free field $\vartheta_{n}$ and

$$
\mathcal{A}_{C}=\int d^{2} x\left[\frac{1}{8 \pi}\left(\left(\partial_{\mu} \varphi \cdot \partial_{\mu} \varphi\right)+\left(\partial_{\mu} \vartheta \cdot \partial_{\mu} \vartheta\right)+\left(\partial_{\mu} \vartheta_{0} \partial_{\mu} \vartheta_{0}\right)\right)+U\left(\varphi, \vartheta, \vartheta_{0}\right)+R_{2} \Phi\right]
$$

with $U\left(\varphi, \phi, \phi_{0}\right)=\mu \sum_{j=1}^{n-1}\left(e^{b\left(h_{j} \cdot \varphi\right)+\mathrm{i} a\left(\eta_{j} \cdot \vartheta\right)+\mathrm{i} c \vartheta_{0}}+e^{-b\left(h_{j+1} \cdot \varphi\right)-\mathrm{i} a\left(\eta_{j} \cdot \vartheta\right)-\mathrm{i} c \vartheta_{0}}\right)$, defines the CFT with central charge:

$$
c=1+(n-1)\left(2+\frac{n(n+1)}{b^{2}}-\frac{n(n-2)}{a^{2}}\right) .
$$

As we see later, the CFT (66) describes the dual representation for coset CFT $\frac{S L(n)}{S L(n-1) U(1)}$. For $n=2$ it coincides with Sine-Liouville CFT (see for example [23])

The primary fields in this CFT (we do not consider here the free field $\vartheta_{n}$ ) are the exponential fields:

$$
V_{A, B, C}=\exp \left((A \cdot \varphi)+\mathrm{i}(B \cdot \phi)+\mathrm{i} C \vartheta_{0}\right)
$$

with dimension

$$
\Delta(A, B, C)=\frac{1}{2}\left(-A^{2}+2(A \cdot Q)+B^{2}-2\left(B \cdot Q_{1}\right)+C^{2}\right) .
$$

In the space of the fields $V_{A, B, C}$ acts the Weyl group $\mathbf{W}$, which is the product of groups $\mathbf{w}_{n}$ and $\mathbf{w}_{n-1}$ of the Lie algebras $\operatorname{sl}(n)$ and $\operatorname{sl}(n-1)$. Here we consider the group $\mathbf{w}_{n}$, which acts as $V_{A, B, C} \rightarrow \mathbf{R}_{\widehat{s}} V_{Q+\widehat{s}(A-Q), B, C}\left(\widehat{s} \subset \mathbf{w}_{n}\right)$ where $\mathbf{R}_{\widehat{s}}$ is the reflection amplitude [42, 41] 2 We normalize our fields by the condition:

$$
\left\langle V_{A, B, C}(x) V_{2 Q-A, 2 Q_{1}-B,-C}(0)\right\rangle=|x|^{-4 \Delta(A, B, C)}
$$

Then the reflection amplitude can be expressed through two point function:

$$
\left.\left\langle V_{Q+\mathbf{a}, Q_{1}+\mathbf{b}, C}(x)\right\rangle V_{Q+\mathbf{a}^{*}, Q_{1}-\mathbf{b},-C}(0)\right\rangle=\mathbf{R}(\mathbf{a}, \mathbf{b}, C)|x|^{-4 \Delta}
$$

where vectors $\mathbf{a}^{*}$ and $\mathbf{a}$ are related by the usual conjugation: $\left(\mathbf{a} \cdot e_{j}\right)=\left(\mathbf{a}^{*} \cdot e_{n-j}\right),\left(\mathbf{a} \cdot h_{j}\right)=$ $-\left(\mathbf{a}^{*} \cdot h_{n+1-j}\right)$, here $e_{j}=h_{j}-h_{j+1}$, are the simple roots of $\operatorname{sl}(n)$.

The function $\mathbf{R}(\mathbf{a}, \mathbf{b}, C)$ in the CFT ([6) can be calculated exactly using the integral relation [27, [28].

$$
\begin{aligned}
\int \mathcal{D}_{n}(x) \prod_{i=1}^{n} \prod_{j=1}^{n+m+2}\left|x_{i}-t_{j}\right|^{2 p_{j}} d^{2 n} x & =\prod_{j=1}^{n+m+2} \frac{\Gamma\left(1+p_{j}\right)}{\Gamma\left(-p_{j}\right)} \prod_{k<J}\left|t_{k}-t_{j}\right|^{2 p_{k}+2 p_{j}+2} \\
& \times \int \mathcal{D}_{m}(y) \prod_{i=1}^{m} \prod_{j=1}^{n+m+2}\left|y_{i}-t_{j}\right|^{-2-2 p_{j}} d^{2 m} y
\end{aligned}
$$

where $\mathcal{D}_{n}(x)=\prod_{i<j}\left|x_{i}-x_{j}\right|^{2}, d^{2 n} x=\frac{1}{n ! \pi^{n}} \prod_{i=1}^{n} d^{2} x_{i}, \quad \sum_{j=1}^{n+m+2} p_{j}=-n-1$; and has the form:

$$
\begin{aligned}
\mathbf{R}(\mathbf{a}, \mathbf{b}, C) & =\prod_{j=1}^{[n / 2]}\left(\frac{\Gamma\left(\frac{1}{2}-b\left(\mathbf{a} \cdot h_{j}\right)+a\left(\mathbf{b} \cdot \eta_{j}\right)+c C\right) \Gamma\left(\frac{1}{2}-b\left(\mathbf{a}^{*} \cdot h_{j}\right)-a\left(\mathbf{b} \cdot \eta_{j}\right)-c C\right)}{\Gamma\left(\frac{1}{2}+b\left(\mathbf{a} \cdot h_{j}\right)-a\left(\mathbf{b} \cdot \eta_{j}\right)-c C\right) \Gamma\left(\frac{1}{2}+b\left(\mathbf{a}^{*} \cdot h_{j}\right)+a\left(\mathbf{b} \cdot \eta_{j}\right)+c C\right)}\right)^{n-2 j+1} \\
& \times\left(\frac{\pi \mu}{b^{2}}\right)^{2(\mathbf{a} \cdot \rho) / b} \prod_{\alpha>0} \frac{\Gamma\left(1+b\left(\mathbf{a} \cdot e_{\alpha}\right)\right) \Gamma\left(1+\frac{1}{b}\left(\mathbf{a} \cdot e_{\alpha}\right)\right)}{\Gamma\left(1-b\left(\mathbf{a} \cdot e_{\alpha}\right)\right) \Gamma\left(1-\frac{1}{b}\left(\mathbf{a} \cdot e_{\alpha}\right)\right)}
\end{aligned}
$$

\footnotetext{
${ }^{2}$ Reflection amplitudes identify the CFTs which admit the free field representation.
} 
where the last product runs over all positive roots. This function corresponds to the maximal reflection $\mathbf{a} \rightarrow-\mathbf{a}^{*}$ and can be represented in the form:

$$
\mathbf{R}(\mathbf{a}, \mathbf{b}, C)=\frac{\mathbf{A}\left(-\mathbf{a}^{*}, \mathbf{b}, C\right)}{\mathbf{A}(\mathbf{a}, \mathbf{b}, C)}
$$

where $\frac{1}{\mathbf{A}(\mathbf{a}, \mathbf{b}, C)}$ is entire function, which includes all products in the denominator of (10).

$$
\begin{aligned}
\mathbf{A}(\mathbf{a}, \mathbf{b}, C) & \left.=\prod_{j=1}^{[n / 2]}\left(\Gamma\left(\frac{1}{2}+b\left(\mathbf{a} \cdot h_{j}\right)-a\left(\mathbf{b} \cdot \eta_{j}\right)-c C\right) \Gamma\left(\frac{1}{2}+b\left(\mathbf{a}^{*} \cdot h_{j}\right)+a\left(\mathbf{b} \cdot \eta_{j}\right)+c C\right)\right)\right)^{n-2 j+1} \\
& \times\left(\frac{\pi \mu}{b^{2}}\right)^{-(\mathbf{a} \cdot \rho) / b} \prod_{\alpha>0} \Gamma\left(1-b\left(\mathbf{a} \cdot e_{\alpha}\right)\right) \Gamma\left(1-\frac{1}{b}\left(\mathbf{a} \cdot e_{\alpha}\right)\right)
\end{aligned}
$$

The general reflection amplitudes $\mathbf{R}_{\widehat{s}}(\mathbf{a}, \mathbf{b}, C)$ are:

$$
\mathbf{R}_{\widehat{s}}(\mathbf{a}, \mathbf{b}, C)=\frac{\mathbf{A}(\widehat{s} \mathbf{a}, \mathbf{b}, C)}{\mathbf{A}(\mathbf{a}, \mathbf{b}, C)} .
$$

These functions play the important role in the analysis of UV asymptotics in perturbed CFTs and in calculation of expectation values of the fields in these theories. In this paper we also use these functions in section 7 to conjecture the dual SM representation for CFT (6) .

\section{Deformed $C P(n-1)$ models with fermion and dual QFT}

The $C P(n-1)$ models are two-dimensional asymptotically free sigma-models on $(n-1)$ complex dimensional Kähler space with an $S U(n)$ invariant metric. As all sigma models with Kähler metric they possess instantons which are the holomorphic functions of world -sheet coordinates - The Kähler metric can be written as $d s^{2}=g_{a b^{*}} d w_{a} d w_{b^{*}}^{*}$ and defines a two form $\kappa=\mathrm{i} g_{a b^{*}} d w_{a} \wedge$ $d w_{b^{*}}^{*}$ This form after proper normalization defines the density of topological charge and is closed (but not exact). It means that locally $\kappa=d \beta$, where $\beta$ is a one form. In the world sheet coordinates this form defines the $U(1)$ gauge field $B$ which can interact with a complex fermion field $\chi$. We call the theory with action:

$$
\mathcal{A}_{d}=\mathcal{A}_{s m}+\int \frac{d^{2} x}{4 \pi} \bar{\chi} \mathrm{i} \gamma_{\mu}\left(\partial_{\mu}+\mathrm{i} B_{\mu}\right) \chi
$$

where $\mathcal{A}_{s m}$ is the action of sigma model, as deformed $C P(n-1)$ models with fermion. After the deformation the $S U(n)$ symmetry of the metric is broken up to $U(1)^{n-1}$. One more $U(1)$ symmetry comes from the fermion interacting with $B$, so the full symmetry of theory is $U(1)^{n}$. We note that the introduction of fermionic field interacting with sigma model cancels the contributions of the fields with a non-zero topological charge and long range interaction in the QFT (14).

To construct the integrable QFT which can be considered as perturbed CFT (6) we have two possibilities. To add the affine pair of fields, or to add the term $\mu_{1} e^{e_{0} \cdot \varphi}$, where $e_{0}$ is the affine root of $\operatorname{sl}(n)$. 
To discuss the relation with sigma model (14) we consider the first possibility. Namely, we add the affine pair (which we denote as $\left.\left\{V_{n}, V_{-n-1}\right\}\right)$. It is convenient to make the orthogonal transformation from $n$ the fields $\left\{\left(\vartheta_{1}, . ., \vartheta_{n-2}\right), \vartheta_{0}, \vartheta_{n}\right\}$ to $n$ fields $\left\{\left(\phi_{1}, . ., \phi_{n-1}\right), \phi_{0}\right\}$ in such way that pairs $\left\{V_{k}, V_{-k-1}\right\}(k=1, . ., n-1)$ (4) transform to

$$
\left\{\mu \exp \left(b\left(h_{k} \cdot \varphi\right)+\mathrm{i} a\left(h_{k} \cdot \phi\right)+\mathrm{i} n^{-1 / 2} \phi_{0}\right), \mu \exp \left(-b\left(h_{-k-1} \cdot \varphi\right)-\mathrm{i} a\left(h_{k} \cdot \phi\right)-\mathrm{i} n^{-1 / 2} \phi_{0}\right)\right\}
$$

and the affine pair $\left\{V_{n}, V_{-n-1}\right\}$ is:

$$
\left\{\mu \exp \left(b\left(h_{n} \cdot \varphi\right)+\mathrm{i} a\left(h_{n} \cdot \phi\right)+\mathrm{i} n^{-1 / 2} \phi_{0}\right), \mu \exp \left(-b\left(h_{1} \cdot \varphi\right)-\mathrm{i} a\left(h_{n} \cdot \phi\right)-\mathrm{i} n^{-1 / 2} \phi_{0}\right)\right\}
$$

The action of our QFT will now have a form:

$$
\mathcal{A}_{s l(n)}^{(1)}=\int d^{2} x\left[\frac{1}{8 \pi}\left(\left(\partial_{\mu} \varphi \cdot \partial_{\mu} \varphi\right)+\left(\partial_{\mu} \phi \cdot \partial_{\mu} \phi\right)+\left(\partial_{\mu} \phi_{0} \partial_{\mu} \phi_{0}\right)\right)+\mathbf{U}\left(\varphi, \phi, \phi_{0}\right)\right]
$$

where $\mathbf{U}\left(\varphi, \phi, \phi_{0}\right)$ can be written in the symmetric form (here and latter $\left.h_{n+1}=h_{1}\right){ }^{3}$

$$
\mathbf{U}\left(\varphi, \phi, \phi_{0}\right)=\sum_{j=1}^{n} \mu\left(e^{b\left(h_{j} \cdot \varphi\right)+\mathrm{i} a\left(h_{j} \cdot \phi\right)+\mathrm{i} n^{-1 / 2} \phi_{0}}+e^{-b\left(h_{j+1} \cdot \varphi\right)-\mathrm{i} a\left(h_{j} \cdot \phi\right)-\mathrm{i} n^{-1 / 2} \phi_{0}}\right)
$$

Besides the discreet symmetries related to permutations of $h_{i}$ the action (15) possesses the $U(1)^{n}$ symmetry associated with the following transformations:

$$
\phi \rightarrow \phi+\frac{2 \pi}{a} \sum_{i=1}^{n-1} e_{i} m_{i}, \quad \phi_{0} \rightarrow \phi_{0}+2 \pi n^{1 / 2} m_{0}, \quad\left(m_{i}, m_{0}\right) \subset Z
$$

In the next section we represent this action in the form suitable for perturbation theory in $b$. In this form the $U(1)^{n}$-symmetry will be obvious.

\section{Action of dual QFT admitting perturbative expan- sion, non-local integrals and scattering theory}

Let us consider the case $n=2$, which is slightly special. Here $h_{1}=\frac{1}{\sqrt{2}}, h_{2}=-\frac{1}{\sqrt{2}}$ and the action has the form:

$$
\mathcal{A}_{s l(2)}^{(1)}=\int d^{2} x\left[\frac{1}{8 \pi}\left(\partial_{\mu} \varphi \partial_{\mu} \varphi+\partial_{\mu} \phi \cdot \partial_{\mu} \phi+\partial_{\mu} \phi_{0} \partial_{\mu} \phi_{0}\right)+\mathbf{U}\left(\varphi, \phi, \phi_{0}\right)\right],
$$

where $\mathbf{U}=2 \mu\left(e^{b \varphi / \sqrt{2}} \cos \left(\frac{\left(a \phi+\phi_{0}\right)}{\sqrt{2}}\right)+e^{-b \varphi / \sqrt{2}} \cos \left(\frac{\left(-a \phi+\phi_{0}\right)}{\sqrt{2}}\right)\right)$. This theory belongs to two parameter family of integrable QFTs [16], with $a_{1}^{2}+a_{2}^{2}-b^{2}=1$ and

$$
\mathbf{U}_{2 p}=2 \mu\left(e^{b \varphi / \sqrt{2}} \cos \left(\frac{\left(a_{1} \phi+a_{2} \phi_{0}\right)}{\sqrt{2}}\right)+e^{-b \varphi / \sqrt{2}} \cos \left(\frac{\left(-a_{1} \phi+a_{2} \phi_{0}\right)}{\sqrt{2}}\right)\right) .
$$

\footnotetext{
${ }^{3}$ The action (15), (16) coincides with the action of $\mathfrak{g l}(n \mid n)$ Toda model [25] after a simple transformation of fields and exclusion of one of them.
} 
Besides the local IM, this theory possesses non-local ones generated by chiral fields

$$
\begin{aligned}
J_{1}^{( \pm)} & =e^{ \pm \mathrm{i} \sqrt{2} / a_{1} \phi}\left( \pm \mathrm{i} a_{1} \partial \phi \pm \mathrm{i} a_{2} \partial \phi_{0}+b \partial \varphi\right), \quad J_{2}^{( \pm)}=e^{ \pm \mathrm{i} \sqrt{2} / a_{2} \phi_{0}}\left( \pm \mathrm{i} a_{1} \partial \phi \pm \mathrm{i} a_{2} \partial \phi_{0}+b \partial \varphi\right), \\
I_{1} & =e^{\sqrt{2} / b \varphi}\left(\mathrm{i} a_{1} \partial \phi+\mathrm{i} a_{2} \partial \phi_{0}+b \partial \varphi\right), \quad I_{2}=e^{-\sqrt{2} / b \varphi}\left(\mathrm{i} a_{1} \partial \phi-\mathrm{i} a_{2} \partial \phi_{0}-b \partial \varphi\right)
\end{aligned}
$$

Non-local integrals of motion generated by currents $J_{1}^{( \pm)}, J_{2}^{( \pm)}$form the quantum group symmetry $S U(2)_{\mathrm{q}_{1}} \otimes S U_{\mathrm{q}_{2}}(2)\left(\mathrm{q}_{j}=\exp \left(\frac{2 \pi \mathrm{i}}{a_{j}^{2}}\right)\right)$ and it is not surprising that scattering matrix of this QFT can be expressed in terms of a direct product of two $S$-matrices of Sine-Gordon model. If we denote the coupling constant in SG model as $\beta_{S G}^{2}=\frac{p}{p+1}$, the scattering matrix of the theory (18) will be

$$
S_{2 p}(\theta)=-S_{p_{1}}^{(S G)}(\theta) \otimes S_{p_{2}}^{(S G)}(\theta)
$$

here $\theta=\theta_{1}-\theta_{2}$ is the relative rapidity of colliding particles and $S_{p_{i}}^{(S G)}(\theta)$ are $S$-matrices of SG-models with $\beta_{i, S G}^{2}=\frac{p_{i}}{p_{i}+1}$ and $p_{i}=a_{i}^{2}$. This fact shows that the deformed $C P(1)$-model with fermion possesses two-parameter family of deformation. In our case $p_{1}=a_{1}^{2}=1+b^{2}$, $p_{2}=1$, and $S$-matrix of the QFT (17) will be the matrix

$$
S=-S_{a^{2}}^{(S G)}(\theta) \otimes S_{1}^{(S G)}(\theta)=-S_{p_{1}}^{(S G)}(\theta) \otimes S_{1}^{(S G)}(\theta)
$$

$S$-matrix $S_{1}^{(S G)}(\theta)$ coincides with $S$-matrix of free fermion theory, but the full scattering matrix is not trivial. We will not discuss in this section the two-parametric deformations of action $\mathcal{A}_{s l(2)}^{(1)}$ but to understand the structure of the particles and the perturbation theory for small $b$, it is convenient to start with the case $n=2$. In this case after application of ColemanMandelstam $2 d$ correspondence [1],[2] between fermions and bosons

$$
\frac{1}{2} \partial_{\mu} \xi \partial_{\mu} \xi \rightarrow \mathrm{i} \bar{\psi} \gamma_{\mu} \partial_{\mu} \psi, \quad \partial_{\mu} \xi \rightarrow \bar{\psi} \gamma_{\mu} \psi, \quad e^{ \pm \mathrm{i} \xi} \rightarrow \bar{\psi}\left(1+\gamma_{5}\right) \psi
$$

we can rewrite the action $\mathcal{A}_{s l(2)}^{(1)}$ (after transformation $\left.\xi_{1}=\frac{1}{\sqrt{2}}\left(a \phi+\phi_{0}\right), \xi_{2}=\frac{1}{\sqrt{2}}\left(-a \phi+\phi_{0}\right)\right)$ as

$$
\mathcal{A}_{s l(2)}^{(1)}=\int\left(L_{F}+L_{F B}+L_{B}\right) d^{2} x
$$

where

$$
\begin{aligned}
L_{F} & =\frac{1}{4 \pi}\left(\sum_{i=1}^{2}\left(\mathrm{i} \bar{\psi}_{i} \gamma_{\mu} \partial_{\mu} \psi_{i}-\frac{b^{2}}{2\left(1+b^{2}\right)}\left(\bar{\psi}_{i} \gamma_{\mu} \psi_{i}\right)^{2}\right)+\frac{b^{2}}{\left(1+b^{2}\right)}\left(\bar{\psi}_{1} \gamma_{\mu} \psi_{1}\right)\left(\bar{\psi}_{2} \gamma_{\mu} \psi_{2}\right)\right), \\
L_{F B} & =\frac{M_{0}}{4 \pi}\left(e^{b \frac{\varphi}{\sqrt{2}}} \bar{\psi}_{1} \psi_{1}+e^{-b \frac{\varphi}{\sqrt{2}}} \bar{\psi}_{2} \psi_{2}\right), \quad L_{B}=\frac{\partial_{\mu} \varphi \partial_{\mu} \varphi}{8 \pi}+\frac{M_{0}^{2}}{4 \pi b^{2}}\left(e^{\sqrt{2} b \varphi}+e^{-\sqrt{2} b \varphi}\right) .
\end{aligned}
$$

The last term in $L_{B}$ is the usual contact counterterm which cancels the divergencies, coming from fermion loops. The particles in this QFT are fermions $\psi_{1}, \psi_{1}^{*} ; \psi_{2}, \psi_{2}^{*}$, with $U(1) \otimes U(1)$ charges $(1,-1) \otimes(1,-1)$. It is easy to check that the perturbation theory in $b$ coincides with small $b$ expansion of $S$-matrix of our theory.

In general case the QFT (15) possesses non-local IM generated by the currents $J_{i}, I_{i} \quad(i=$ $1, . ., n)$ :

$$
\begin{aligned}
& J_{i}=\exp \left(\frac{\mathrm{i}}{a}\left(e_{i} \cdot \phi\right)\right)\left(\mathrm{i} a\left(h_{i} \cdot \partial \phi\right)+b\left(h_{i+1} \cdot \partial \varphi\right)+\mathrm{i} \partial \phi_{0} n^{-1 / 2}\right), \\
& I_{i}=\exp \left(\frac{1}{b}\left(e_{\mathrm{i}} \cdot \varphi\right)\right)\left(\mathrm{i} a\left(h_{i} \cdot \partial \phi\right)+b\left(h_{i} \cdot \partial \varphi\right)+\mathrm{i} \partial \phi_{0} n^{-1 / 2}\right)
\end{aligned}
$$


where $e_{n}=h_{n}-h_{1}$ and $h_{n+1}=h_{1}$. In appendix $A$ we provide the examples of local integrals of motion.

The IM corresponding to currents $J_{i}$ generate $S U(n)_{\mathrm{q}}$ (with q $=\exp \left(\frac{2 \pi \mathrm{i}}{a^{2}}\right)$ ) symmetry of the scattering theory of this QFT. The solution of Yang-Baxter equations with this symmetry was constructed by Cherednik [32]. To understand better the content of particles and spectrum of this theory, it is useful to use again the Coleman-Mandelstam $2 d$ correspondence [1, [2] between fermion and bosons (22). We introduce the fields

$$
\xi_{i}=a\left(h_{i} \cdot \phi\right)+\phi_{0} n^{-1 / 2}
$$

and projectors $\gamma_{+}, \gamma_{-}: \gamma_{ \pm}=\frac{1}{2}\left(1 \pm \gamma_{5}\right)$. Then the action (15) after application of ColemanMandelstam rules can be rewritten in the form:

$$
\mathcal{A}_{s l(n)}^{(1)}=\int d^{2} x\left(L_{F}+L_{B}+L_{F B}\right)
$$

where:

$$
\begin{aligned}
L_{F} & =\frac{1}{4 \pi}\left(\sum_{i=1}^{n}\left(\mathrm{i} \bar{\psi}_{i} \gamma_{\mu} \partial_{\mu} \psi_{i}-g_{1}\left(\bar{\psi}_{i} \gamma_{\mu} \psi_{i}\right)^{2}\right)+g_{2} \sum_{i \neq j}^{n}\left(\bar{\psi}_{i} \gamma_{\mu} \psi_{i}\right)\left(\bar{\psi}_{j} \gamma_{\mu} \psi_{j}\right)\right), \\
L_{F B} & =\frac{M_{0}}{4 \pi} \sum_{j=1}^{n}\left(e^{b\left(h_{j} \cdot \varphi\right)} \bar{\psi}_{i} \gamma_{+} \psi_{i}+e^{-b\left(h_{j}+1 \cdot \varphi\right)} \bar{\psi}_{i} \gamma_{-} \psi_{i},\right) \\
L_{B} & =\frac{1}{8 \pi}\left(\left(\partial_{\mu} \varphi \cdot \partial_{\mu} \varphi\right)+\frac{2 M_{0}^{2}}{b^{2}} \sum_{j=0}^{n-1} e^{b\left(e_{j} \cdot \varphi\right)}\right), \quad g_{1}=\frac{(n-1) b^{2}}{n\left(1+b^{2}\right)}, \quad g_{2}=\frac{b^{2}}{n\left(1+b^{2}\right)} .
\end{aligned}
$$

The last term in $L_{B}$, which has a form of $s l_{n}$ Toda potential ( $e_{0}$ is the affine root of $s l_{n}$ ), is the usual contact counterterm which cancels the divergencies, coming from fermion loops. This QFT has a broken $\mathbf{P}$ symmetry but possesses $\mathbf{P T}$ and $\mathbf{C}$ symmetries. For small $b$ it has $2 n$ particles $\psi_{i}, \psi_{i}^{*}$ with mass $M$, and $(n-1)$ scalar particles $\varphi_{i}$ which can be considered as bound states of Fermi particles with masses $M_{j}=2 M \sin \left(\frac{\pi j}{n}\right)+O\left(b^{2}\right)$. As we see later, for finite $b$ they disappear from spectrum.

The scattering theory of basic particles $\psi_{i} \rightarrow i,, \psi_{i}^{*} \rightarrow \bar{i}$ has $U(1)^{n}$ symmetry. For $n>2$ the $S$-matrix of these particles is also restricted by $S U(n)_{\mathrm{q}}$ symmetry and has no backward scattering amplitudes of particles and antiparticles. It also should satisfy PT, C, and crossing symmetries:

$$
S_{j_{1} j_{2}}^{i_{1} i_{2}}(\theta)=S_{i_{2} i_{1}}^{j_{2} j_{1}}(\theta), \quad S_{j_{1} j_{2}}^{i_{1} i_{2}}(\theta)=S_{\bar{j}_{1} \bar{j}_{2}}^{\bar{i}_{1} \bar{i}_{2}}(\theta), \quad S_{j_{1} j_{2}}^{i_{1} i_{2}}(\theta)=S_{j_{2} \bar{i}_{2}}^{i_{2} \bar{j}_{2}}(\mathrm{i} \pi-\theta) .
$$

It is convenient to write these amplitudes in the form:

$$
\begin{aligned}
& S_{i i}^{i i}(\theta)=-F(\theta), \quad S_{i j}^{i j}(\theta)=-F(\theta) \frac{\sinh \left(\frac{n}{2} \lambda \theta\right)}{\sinh \left(\frac{n}{2} \lambda\left(\theta-i \frac{2 \pi}{n}\right)\right.}, \\
& S_{j i}^{i j}(\theta)=-F(\theta) \frac{\sin (\pi \lambda) \exp \left(\mathrm{i} \kappa_{i, j} \lambda \theta\right)}{\sinh \left(\frac{n}{2} \lambda\left(\theta-\mathrm{i} \frac{2 \pi}{n}\right)\right.},
\end{aligned}
$$

where $i \neq j$ and $\kappa_{i, j}=i-j-\frac{n}{2} \operatorname{sgn}(i-j)$. The function $F(\theta)$ satisfies the unitarity and crossing symmetry conditions:

$$
F(\theta) F(-\theta)=1, \quad F(\mathrm{i} \pi-\theta) F(\mathrm{i} \pi+\theta)=\frac{\sinh \left(\frac{n}{2} \lambda(\theta+\mathrm{i} \pi)\right) \sinh \left(\frac{n}{2} \lambda(-\theta+\mathrm{i} \pi)\right)}{\sinh \left(\frac{n}{2} \lambda\left(\theta+\mathrm{i} \pi \frac{n-2}{n}\right)\right) \sinh \left(\frac{n}{2} \lambda\left(-\theta+\mathrm{i} \pi \frac{n-2}{n}\right)\right)}
$$


It is convenient to introduce parameter $p$ :

$$
p=\frac{1}{\lambda}
$$

Then the solution to the equations (27) can be written as:

$$
F_{n}(\theta)=\exp \left(\mathrm{i} \delta_{n, p}(\theta)\right)=\exp \left(\mathrm{i} \int_{-\infty}^{\infty} \frac{d \omega}{\omega} \frac{\sinh \left(\frac{\pi(n-1) \omega}{n}\right) \sinh \left(\frac{\pi(p-1) \omega}{n}\right)}{\sinh (\pi \omega) \sinh \left(\frac{\pi p \omega}{n}\right)} \sin (\omega \theta)\right)
$$

We have a one parameter scattering theory. To connect it with QFT (25), (15) we should find a relation between parameter $\lambda$ (or $p$ ) and the coupling constant $b$. To solve this problem we need to perform the non-perturbative Bethe Ansatz (BA) analysis.

\section{Bethe Ansatz analysis}

To find the relations between the parameters of the action and the scattering parameters it is important to calculate the observables using both approaches. Our QFT possesses $U(1)^{n}$ symmetry generated by the charges $Q_{j}=\int \psi_{j} \psi_{j}^{*} d x$. One can add to the Hamiltonian of our QFT the terms $-A_{i} Q_{i}$ with different parameters $A_{i}$ (chemical potentials). The simplest way to calculate the ground state energy using the BA approach is to consider the configurations of the chemical potentials $A_{i}$ which lead to the condensation of particles which have the simple scattering amplitudes, namely, the pure phases. It is useful to consider the case when only one kind of particles is condensed. This configuration corresponds to $A_{1}=A$ and all other $A_{i}=0$. In the case for $A>>M$ we can neglect in the action (24) all terms containing the mass parameter $M_{0}$ and there only particle $\psi_{1}$ will condense. It means that for calculation of density of ground state energy (GSE) we can put all $\psi_{i}$ with $i>1$ equal to zero. The action in this case coincides with the action of massless Thirring model:

$$
\mathcal{A}_{M T M}=\frac{1}{4 \pi} \int d^{2} x\left[\mathrm{i} \bar{\psi}_{1} \gamma_{\mu} \partial_{\mu} \psi_{1}-\frac{(n-1) b^{2}}{n\left(1+b^{2}\right)}\left(\bar{\psi}_{1} \gamma_{\mu} \psi_{1}\right)^{2}\right] \text {. }
$$

The GSE of massless Thirring model with coupling constant $g=-\frac{(n-1) b^{2}}{n\left(1+b^{2}\right)}$ is well known and is given by

$$
\mathcal{E}_{M T M}(A)=-\frac{1}{2 \pi}(1+g)^{-1}=-\frac{n\left(1+b^{2}\right)}{2 \pi\left(n+b^{2}\right)} .
$$

We calculate now the same value from the BA approach. For $A>M$ one has the sea of particles $\psi_{1}(\theta)$ which fill all possible states inside some "Fermi interval" $-B<\theta<B$. The distribution of the particles $\epsilon(\theta)$ within this interval is determined by their scattering amplitude $S_{11}^{11}(\theta)=-F_{n}(\theta)$. The specific ground state energy has the form

$$
\mathcal{E}(A)-\mathcal{E}(0)=-\frac{M}{2 \pi} \int_{-B}^{B} \cosh (\theta) \epsilon(\theta) d \theta
$$

where the non-negative function $\epsilon(\theta)$ satisfies the BA equation, inside the interval $-B<\theta<B$,

$$
\int_{-B}^{B} \widetilde{K}\left(\theta-\theta^{\prime}\right) \epsilon\left(\theta^{\prime}\right) d \theta^{\prime}=A-M \cosh \theta
$$


and the parameter $B$ is determined by the boundary condition $\epsilon( \pm B)=0$.

The kernel $\widetilde{K}(\theta)$ in this equation is related to $\psi_{1} \psi_{1}$ scattering phase $\delta_{n, p}(\theta)(28)$

$$
\widetilde{K}(\theta)=\delta(\theta)-\frac{1}{2 \pi \mathrm{i}} \frac{d}{d \theta} \log \left(F_{n}(\theta)\right)=\delta(\theta)-\frac{1}{2 \pi} \frac{d}{d \theta} \delta_{n, p}(\theta) .
$$

The Fourier transform $K(\omega)$ of this kernel has the form

$$
K(\omega)=\frac{\sinh \left(\frac{\pi \omega}{n}\right) \sinh \left(\frac{\pi(p+n-1) \omega}{n}\right)}{\sinh (\pi \omega) \sinh \left(\frac{\pi p \omega}{n}\right)} .
$$

The main term of asymptotics of the function $\mathcal{E}(A \rightarrow \infty)$ can be expressed explicitly through the kernel $K(\omega)$ by the relation [17]

$$
\mathcal{E}(A \rightarrow \infty)=-\frac{A^{2}}{2 \pi K(0)}=-\frac{n p A^{2}}{2 \pi(p+n-1)} .
$$

Comparing the Eqs (29) and (34), we find that

$$
p=\frac{1}{\lambda}=\left(1+b^{2}\right)
$$

in agreement with perturbation theory. We see that at $b \rightarrow \infty, \lambda \rightarrow 0$ and $S$-matrix (26) tends to the scattering matrix of $C P(n-1)$ model with fermion [33]. So it is natural to call our QFT as the dual theory to deformed $C P(n-1)$ model with fermion.

The term $\mathcal{E}(0)$ in Eq. (30) is the bulk vacuum energy of QFT (24). It can be expressed trough the kernel $K(\omega)$ by the relation

$$
\mathcal{E}(0)=-\frac{M^{2}}{8}\left(\left.K(\omega) \cosh \left(\frac{\pi \omega}{2}\right)\right|_{\omega=\mathrm{i}}\right)^{-1}=\frac{M^{2} \sin \left(\frac{\pi p}{n}\right)}{4 \sin \left(\frac{\pi}{n}\right) \sin \left(\frac{\pi(p-1)}{n}\right)} .
$$

For small $b$ the bulk vacuum energy will be

$$
\mathcal{E}(0)=\frac{n M^{2}}{4 \pi b^{2}}+\frac{M^{2}}{4} \cot \left(\frac{\pi}{n}\right)+O\left(b^{2}\right),
$$

the first term where is the contribution of Toda term in Eq.(25). The second term comes from the renormalization of $M=M_{0}\left(b^{2}\right)$ and from the contribution of the vacuum energies of $n$ free fermions and $(n-1)$ bosonic Toda particles with masses $M_{k}=2 M \sin (\pi k / n),(k=1, . .,[n / 2])$. The second contribution can be easily calculated and is given by

$$
\begin{aligned}
\delta \mathcal{E}(0) & =-\frac{M^{2}}{4 \pi}\left(\sum_{k=1}^{[n / 2]} 4 \sin (\pi k / n)^{2} \log \left(\frac{M_{k}^{2}}{M^{2}}\right)-\left(1+(-1)^{n}\right) \log \left(\frac{M_{n / 2}^{2}}{M^{2}}\right)\right) \\
& =-\frac{M^{2}}{4 \pi}\left(\sum_{k=1}^{[n / 2]} 4 \sin (\pi k / n)^{2} \log \left(4 \sin (\pi k / n)^{2}\right)-\left(1+(-1)^{n}\right) \log (4)\right) .
\end{aligned}
$$


The exact relation between the physical mass $M$ and parameter $M_{0}$ can be calculated by the BA methods [38, 39] and has the form:

$$
M=M_{0} \frac{\Gamma\left(\frac{p}{n}\right)}{\Gamma\left(\frac{1}{n}\right) \Gamma\left(\frac{p+n-1}{n}\right)}=M_{0}\left(1+\frac{\left(\psi\left(\frac{1}{n}\right)-\psi(1)\right)}{n} b^{2}+O\left(b^{4}\right)\right)
$$

It is easy to derive from Eqs.(37)-(39) the agreement with the perturbation theory and the sum rule:

$$
\begin{aligned}
& \frac{1}{\pi}\left(\sum_{k=1}^{[n / 2]} \sin (\pi k / n)^{2} \log \left(4 \sin (\pi k / n)^{2}\right)-\frac{1}{4}\left(1+(-1)^{n}\right) \log (4)\right) \\
& =-\left(\frac{\cot \left(\frac{\pi}{n}\right)}{4}+\frac{2\left(\psi\left(\frac{1}{n}\right)-\psi(1)\right)}{n}\right)
\end{aligned}
$$

here $\psi(z)=\Gamma^{\prime}(z) / \Gamma(z)$. We note that QFT (25) provides us by example of theory which has no UV divergences in perturbation theory in $b$. This nice property is general for QFTs possessing the "nice" duality with asymptotically free SMs.

For $b \rightarrow \infty$ we, represent $\mathcal{E}(0)=\frac{M^{2} \sin \left(\frac{\pi p}{n}\right)}{4 \sin \left(\frac{\pi}{n}\right) \sin \left(\frac{\pi(p-1)}{n}\right)}=\frac{M^{2}}{4}\left(\cot (\pi / n)+\cot \left(\pi b^{2} / n\right)\right)$. The second term oscillates around zero with period $b^{2} / n$. It is reasonable to define the bulk ground state energy as the meaning value over some interval much bigger then this period. With this definition we derive that bulk ground state energy of $C P(n-1)$ model with fermion is given by: $\mathcal{E}(0)=\frac{M^{2}}{4} \cot (\pi / n)$, what agrees with large $n$ expansion.

The integral BA equation can be studied using the generalized Winner-Hopf method [30]. We can go further in the $\operatorname{UV}\left(\frac{A}{M}>>1\right)$ analysis of the function $\mathcal{E}(A)$. In particular, the UV corrections to Eq.(34) have the form $\left(\frac{M}{A}\right)^{2 \nu_{j}}$, where the exponents $\nu_{j}$ are defined (with the factor $1 / \mathrm{i}$ ) by the zeroes of the kernel (33) in the upper half-plane. For integer $n$ the zeroes at $\omega_{k}=\mathrm{i} n k$ are cancelled by the denominator of the kernel (this phenomenon is related with the cancellation of instanton contribution by the fermion in Eq (14)). The zeroes at $\omega_{j}=\mathrm{i} \frac{n j}{(p+n-1)},\left(\nu_{j}=\frac{n j}{(p+n-1)}\right)$ we call the perturbative ones. For small $b$, the corresponding exponents $2 \nu_{j}=\frac{2 n j}{\left(b^{2}+n\right)}$ appear after summation of logarithms in the perturbative series. As a result the UV expansion has the form

$$
\mathcal{E}(A)=-\frac{n p A^{2}}{2 \pi(p+n-1)}\left(1+\sum_{k}^{\infty} d_{k}\left(\frac{M}{A}\right)^{\frac{2 n k}{(p+n-1)}}\right)
$$

All coefficients $d_{k}$ can be expressed trough the residues of the function $\rho(\omega)=N(\omega) / N(-\omega)$, where $N(\omega)$ is the function analytical in the lower half plane which factorizes the kernel $K(\omega)=$ $(N(\omega) N(-\omega))^{-1}$. In particular, the first coefficient

$$
d_{1}=-\frac{2(p+n-1) \Gamma\left(\frac{-1}{p+n-1}\right) \Gamma\left(\frac{2 p+3 n-2}{p+n-1}\right) \Gamma\left(\frac{-p}{p+n-1}\right)}{(p+n-2) \Gamma\left(\frac{p+n}{p+n-1}\right) \Gamma\left(\frac{2 p+n-2}{p+n-1}\right) \Gamma\left(\frac{p}{p+n-1}\right)}\left(\frac{\Gamma\left(\frac{p+2 n-1}{n}\right) \Gamma\left(\frac{n+1}{n}\right)}{2 \Gamma\left(\frac{p+n}{n}\right)}\right)^{\frac{2 n}{p+n-1}}
$$

The function $\mathcal{E}(A)$ simplifies drastically in the scaling limit $b^{2}=p-1>>1, \log \left(\frac{A}{M}\right)>>1$ with $\log \left(\frac{A}{M}\right) / p$ fixed. This approximation is useful for the analysis of the UV behavior in dual 
(sigma-model) representation of our QFT. The function $\mathcal{E}(A)$ in this approximation (up to $\frac{1}{p^{2}}$ ) is

$$
\mathcal{E}(A)=-\frac{n A^{2}}{2 \pi}\left(1-\frac{(n-1)}{p+n-1}\left(\frac{1+q^{2}}{1-q^{2}}+\frac{2(n-1) q^{2} \log \left(\frac{p\left(1-q^{2}\right)}{n e^{1 / 2}}\right)}{p\left(1-q^{2}\right)^{2}}\right)\right)
$$

here $q=\left(\frac{M \Gamma\left(\frac{n+1}{n}\right)}{2 A}\right)^{\frac{n}{p+n-1}}$, and the omitted terms are $O\left(1 / p^{3}\right)$. In the limit $b^{2}=p-1 \rightarrow \infty$, we derive the known result [48] for $C P(n-1)$ with fermion

$$
\mathcal{E}(A)=-\frac{n A^{2}}{2 \pi}\left(1-\frac{n-1}{n \log (A / M)}-\frac{(n-1)^{2} \log (\log (A / M))+n(n-1) D_{n}}{n^{2} \log (A / M)^{2}}+. .\right)
$$

where $D_{n}=\log \left(\Gamma\left(\frac{n+1}{n}\right)\right)-\frac{\log (2)}{n}-\frac{(n-3)}{2 n}$.

We note that UV behavior of function (40), (41) is not characteristic for asymptotically free sigma models. In these models GSE $\mathcal{E}(A) / A^{2}$ usually tends to infinity in the scaling limit. A different behavior, depending on the chose of parameters $A_{i}$, appears in the models which have several $U(1)$ symmetries (see [16]). Here we show that similar phenomenon takes place in our QFT. The GSE (41) is related with $U(1)$ associated with fermion $\chi$ in the action (14) or with field $\phi_{0}$ in the action (15).

As it was noted before the BA equations for the GSE in the fields $A_{i}$ is easy to study when the scattering amplitudes of condensed particles are pure phases. The corresponding situation is realized when we take $A_{1}=A$, and $A_{2}=-A$. In this case the scattering amplitudes are given by

$$
S_{11}^{11}(\theta)=S_{\overline{22}}^{\overline{22}}(\theta)=\exp \left(\mathrm{i} \delta_{n, p}(\theta)\right), \quad S_{1 \overline{2}}^{1 \overline{2}}(\theta)=S_{\overline{2} 1}^{\overline{2} 1}(\theta)=\exp \left(\mathrm{i} \delta_{n, p}^{(1)}(\theta)\right) .
$$

The function $\delta_{n, p}(\theta)$ is given by Eq.(28) and

$$
\delta_{n, p}^{(1)}(\theta)=\int_{-\infty}^{\infty} \frac{d \omega \sinh \left(\frac{\pi \omega}{n}\right) \sinh \left(\frac{\pi(p-1) \omega}{n}\right)}{\omega \sinh (\pi \omega) \sinh \left(\frac{\pi p \omega}{n}\right)} \sin (\omega \theta)
$$

With these data we can write the system of BA integral equations for the calculation of function $\mathcal{E}_{-}(A)$

$$
\mathcal{E}_{-}(A)-\mathcal{E}(0)=-\frac{M}{2 \pi}\left(\int_{-B}^{B} \cosh (\theta) \epsilon_{1}(\theta) d \theta+\int_{-B}^{B} \cosh (\theta) \epsilon_{2}(\theta) d \theta\right)
$$

where the non-negative functions $\epsilon_{1}(\theta), \epsilon_{2}(\theta)$ satisfy inside the interval $-B<\theta<B$, the BA equations

$$
\begin{aligned}
& \int_{-B}^{B} \widetilde{K}_{1}\left(\theta-\theta^{\prime}\right) \epsilon_{1}\left(\theta^{\prime}\right) d \theta^{\prime}+\int_{-B}^{B} \widetilde{K}_{2}\left(\theta-\theta^{\prime}\right) \epsilon_{2}\left(\theta^{\prime}\right) d \theta^{\prime}=A-M \cosh \theta ; \\
& \int_{-B}^{B} \widetilde{K}_{1}\left(\theta-\theta^{\prime}\right) \epsilon_{2}\left(\theta^{\prime}\right) d \theta^{\prime}+\int_{-B}^{B} \widetilde{K}_{2}\left(\theta-\theta^{\prime}\right) \epsilon_{1}\left(\theta^{\prime}\right) d \theta^{\prime}=A-M \cosh \theta
\end{aligned}
$$

and the parameter $B$ is determined by the boundary condition $\epsilon_{1,2}( \pm B)=0$. 
The kernels $\widetilde{K}_{1,2}(\theta)$ in this equation are

$$
\widetilde{K}_{1}(\theta)=\widetilde{K}(\theta)=\delta(\theta)-\frac{1}{2 \pi} \frac{d}{d \theta} \delta_{n, p}(\theta), \quad \widetilde{K}_{2}(\theta)=-\frac{1}{2 \pi} \frac{d}{d \theta} \delta_{n, p}^{(1)}(\theta) .
$$

The Fourier transforms $K_{1,2}(\omega)$ of these kernels have the form

$$
K_{1}(\omega)=K(\omega), \quad K_{2}(\omega)=-\frac{\sinh \left(\frac{\pi \omega}{n}\right) \sinh \left(\frac{\pi(p-1) \omega}{n}\right)}{\sinh (\pi \omega) \sinh \left(\frac{\pi p \omega}{n}\right)}
$$

The eqs.(42) are symmetric with respect to $\epsilon_{1}(\theta) \leftrightarrow \epsilon_{2}(\theta)$. It means that we can reduce them to one equation with the kernel

$$
K_{0}(\omega)=K(\omega)+K_{2}(\omega)=\frac{\sinh \left(\frac{\pi \omega}{n}\right) \cosh \left(\frac{\pi(p+n / 2-1) \omega}{n}\right)}{\cosh \left(\frac{\pi \omega}{2}\right) \sinh \left(\frac{\pi p \omega}{n}\right)} .
$$

This kernel $K_{0}(\omega)$ coincides exactly with the kernel which appears in the BA equations for the deformed $O(n+2)$ sigma-model. It means that function $\mathcal{E}_{-}(A)-\mathcal{E}(0)$ is equal twice the function $\mathcal{E}_{O(n+2)}(A)-\mathcal{E}(0)$ for deformed $O(n+2)$ sigma-model and possesses all characteristic properties for asymptotically free sigma models. This function was studied in [22] and in the scaling limit together with the first correction $O(1 / p \log p)$ is

$$
\mathcal{E}_{-}(A)=2 \mathcal{E}_{O(n+2)}(A)=-\frac{p A^{2}}{\pi} \frac{1-q}{1+q}\left(1+\frac{2}{p} \frac{q \log \left(\frac{\left(1-q^{2}\right) p}{n}\right)}{1-q^{2}}+. .\right)
$$

where $q=\left(\frac{M}{A} \Gamma\left(\frac{n+1}{n}\right)\right)^{\frac{n}{p}}$. The first term $-\frac{p A^{2}}{\pi} \frac{1-q}{1+q}=-\frac{p A^{2}}{\pi} \tanh \left(\frac{n}{2 p} \log \left(\frac{M}{A}\right)\right)$ corresponds to the one loop sigma-model metric, whereas the next term appears in the two-loop approximation.

\section{Sigma models, Ricci flow, integrability and observ- ables}

In this section we consider the relation between integrable QFTs, scattering theory data, classically integrable SMs and Ricci flow equations. Integrable QFTs possess factorized scattering theory and their $S$-matrices satisfy Yang-Baxter (YB) equations. When these QFTs have the dual SM representation, the corresponding SMs should be also integrable at least classically. These SMs are called YB SMs [19],[20]. It is very natural that these objects are associated with names of Yang and Baxter. The scattering matrices in the QFTs, which have the dual SM representation are the deformations of $S$-matrices of SMs on the symmetric spaces (sometimes with additional degrees of freedom which are necessary for quantum integrability). The YB SMs are also the models on the deformed symmetric spaces. One can expect a correspondence between the parameters of deformation in both theories. To find it, we compare the observables calculated from scattering theory data and from SMs.

To realize this problem, it is very important to find a relation between YB SMs and solutions of Ricci flow equations. In many cases this relation is rather non-trivial and includes the diffeomorphisms of metrics depending on the RG time (logarithm of the scale). In this 
section we discuss how sometimes this problem can be avoided. We note that in general case the $S$-matrices, corresponding to deformed symmetric spaces, do not have the values of the deformation parameters where they are equal to identity matrice. In these cases the representation of QFT in terms of fields does not appear to be very useful, because there is no weak coupling region. However, even in these cases such representation can be used for construction of non-local integrals of motion, which help to reconstruct the $S$-matrix.

To start with, we consider the case where we have the exact correspondence between QFT, factorized scattering theory (20), and SM [16. All these objects depend on two parameters $p_{1}, p_{2}$ and describe a two-parameter deformation of $S U(2)$ principle chiral field. To write the action of this SM, it is useful to introduce the coordinates for $g \subset S U(2)$

$$
g=\left(\begin{array}{cc}
e^{\mathrm{i} \beta_{1}+\mathrm{i} \beta_{2}} \cos \theta & e^{\mathrm{i} \beta_{1}-\mathrm{i} \beta_{2}} \sin \theta \\
e^{-\mathrm{i} \beta_{1}+\mathrm{i} \beta_{2}} \sin \theta & e^{-\mathrm{i} \beta_{1}-\mathrm{i} \beta_{2}} \cos \theta
\end{array}\right)
$$

Then action is

$$
\mathcal{A}(S U(2))=\frac{\sigma}{4 \pi} \int d^{2} x\left(\frac{u(t) \operatorname{tr}\left(\partial_{\mu} g \partial_{\mu} g^{-1}\right)-2 l(t)\left(L_{\mu}^{3}\right)^{2}-2 r(t)\left(R_{\mu}^{3}\right)^{2}}{\left(a(t)^{2}-b(t)^{2}\left(\operatorname{tr}\left(g \tau_{3} g^{-1} \tau_{3}\right)\right)\right.}\right)
$$

where $L_{\mu}^{a}=\frac{1}{2} \operatorname{tr}\left(\left(\partial_{\mu} g g^{-1} \tau_{a}\right)\right), R_{\mu}^{a}=\frac{1}{2} \operatorname{tr}\left(\left(\partial_{\mu} g^{-1} g \tau_{a}\right)\right), \sigma=\frac{4 p_{1} p_{2}}{\left(p_{1}+p_{2}\right)}$,

$$
\begin{aligned}
u(t) & =\tau, \quad l(t)+r(t)=\tau\left(1+\sqrt{\left(1-\tau^{2}\right)\left(1-\mathrm{k}^{2} \tau^{2}\right)}\right), \\
l(t)-r(t) & =\mathrm{k} \tau^{2}, \quad a(t)+b(t)=\sqrt{1-\mathrm{k}^{2} \tau^{2}}, \quad a(t)-b(t)=\sqrt{1-\tau^{2}},
\end{aligned}
$$

and parameter .

$$
\mathrm{k}=\frac{p_{1}-p_{2}}{p_{1}+p_{2}}
$$

The function $\tau(t)$ which was denoted in [16] as $\tanh \xi$, is defined by the relation

$$
\left(\frac{1-\tau}{1+\tau}\right)\left(\frac{1-\mathrm{k} \tau}{1+\mathrm{k} \tau}\right)^{\mathrm{k}}=q^{2}(t), \quad q(t)=\exp \left(\frac{2\left(t-t_{0}\right)}{\left(p_{1}+p_{2}\right)}\right)
$$

or $\xi-\frac{\mathrm{k}}{2} \log \left(\frac{1-\mathrm{k} \xi}{1+\mathrm{k} \xi}\right)=\frac{2\left(t_{0}-t\right)}{\left(p_{1}+p_{2}\right)}$.

The metric (45) in the coordinates $\chi_{1}=\frac{\beta_{1}+\beta_{2}}{\sqrt{2}}, \chi_{2}=\frac{\beta_{1}-\beta_{2}}{\sqrt{2}}$ has the form

$$
\begin{aligned}
d s^{2} & =\sigma \frac{u(d \theta)^{2}+(u+r+l \cos 2 \theta)\left(d \chi_{1}\right)^{2}+(u+l+r \cos 2 \theta)\left(d \chi_{2}\right)^{2}}{a^{2}-b^{2} \cos ^{2} 2 \theta} \\
& +\sigma \frac{2(u+l+r) \cos 2 \theta d \chi_{1} d \chi_{2}}{a^{2}-b^{2} \cos ^{2} 2 \theta} .
\end{aligned}
$$

With this action, we can calculate the observables [16], The metric (45) possesses two $U(1)$ symmetries. We introduce the fields $A_{j}$, i.e. $\partial_{0} \chi_{j} \rightarrow\left(\partial_{0}+\mathrm{i} A_{j}\right) \chi_{j}$. To compare the GSE, which is defined as the minimum of the action of SM, with that derived in the previous section from $\mathrm{BA}$, we consider the same configurations of fields (chemical potentials) $A_{j}: A_{1}=A, A_{2}=0$ and $A_{1}=-A_{2}=A$. The field $A$ introduces the scale in our QFT. We put

$$
t_{0}-t=\log \frac{A}{M}
$$


For the first configuration the minimum of the action corresponds to $\theta=0$ and we have

$$
\mathcal{E}=-\frac{\sigma A^{2}(u+r+l)}{4 \pi\left(a^{2}-b^{2}\right)}=-\frac{2 \sigma A^{2} \tau(A)}{4 \pi} .
$$

For the second the minimum of the action corresponds to $\theta=\frac{\pi}{4}$ and

$$
\mathcal{E}_{-}=-\frac{2 \sigma A^{2}(u+r)}{4 \pi a^{2}}=-\frac{\sigma A^{2} \tau(A)}{4 \pi}\left(1+\sqrt{\left(1-\tau^{2}\right)\left(1-\mathrm{k}^{2} \tau^{2}\right)}-\mathrm{k} \tau^{2}\right)^{-1}
$$

It is useful to compare the metric (45) with integrable metric [19], which also depends on two parameters $\alpha$ and $\zeta$ and can be written for arbitrary Lie group $G$. This action can be written using the operator $R$ acting on the generators $T_{a}$ of Lie algebra $G$ as follows: it multiplies the generators corresponding to positive roots by $\mathrm{i}$, to negative roots by $-\mathrm{i}$ and annihilates Cartan subalgebra. Let $\mathcal{R}_{a}^{b}$ is defined as $R\left(T_{a}\right)=\mathcal{R}_{a}^{b} T_{b}$ and $A(g)$ is the matrix of group $G$ in the adjoin representation. We denote $J_{a, \mu}=\operatorname{tr}\left(g^{-1} \partial_{\mu} g T_{a}\right)$

$$
\mathcal{A}_{G}^{\prime}=\frac{\delta^{\mu, \nu}+\varepsilon^{\mu, \nu}}{4 \pi} \Sigma(G) \tau \int d^{2} x\left(J_{a, \mu}\left(I+\mathrm{i} \alpha \mathcal{R}+\mathrm{i} \zeta A(g) \mathcal{R} A(g)^{-1}\right)_{a, b}^{-1} J_{a, \nu}\right) .
$$

For the case of $G=S U(2), \mathcal{R}=\left(\begin{array}{ccc}0 & 1 & 0 \\ -1 & 0 & 0 \\ 0 & 0 & 0\end{array}\right), \Sigma(G)=\sigma \tau$,

$$
A(g) \mathcal{R} A(g)^{-1}=\left(\begin{array}{ccc}
0 & \cos 2 \theta & -\sin 2 \beta_{1} \sin 2 \theta \\
-\cos 2 \theta & 0 & \cos 2 \beta_{1} \sin 2 \theta \\
\sin 2 \beta_{1} \sin 2 \theta & -\cos 2 \beta_{1} \sin 2 \theta & 0
\end{array}\right)
$$

and the metric $d s^{2}$ in the coordinates $\theta, \chi_{1}, \chi_{2}$ is

$$
\begin{aligned}
& \sigma \tau\left(\frac{d \theta^{2}+2 \cos ^{2} \theta\left(1-(\alpha+\zeta)^{2} \cos ^{2} \theta\right) d \chi_{1}^{2}+2 \sin ^{2} \theta\left(1-(\alpha-\zeta)^{2} \sin ^{2} \theta\right) d \chi_{2}^{2}}{1-\alpha^{2}-\zeta^{2}-2 \alpha \zeta \cos 2 \theta}\right) \\
& +\sigma \tau\left(\frac{\left(\zeta^{2}-\alpha^{2}\right) \sin ^{2} 2 \theta d \chi_{1} d \chi_{2}}{1-\alpha^{2}-\zeta^{2}-2 \alpha \zeta \cos 2 \theta}\right) .
\end{aligned}
$$

The metrics (49) and (153) are rather different. However, if we take

$$
\alpha=\frac{1}{2}(1+\mathrm{k}) \tau, \zeta=\frac{1}{2}(1-\mathrm{k}) \tau
$$

the observables calculated with these metrics coincide. For the first configuration the minimum of action (53) corresponds to $\theta=0$ and is

$$
\mathcal{E}(A)=-\frac{2 \sigma A^{2} \tau(A)}{4 \pi}
$$

For other configuration, the position of the minimum is less trivial

$$
\cos ^{2} \theta_{-}=\frac{1-\left(\zeta^{2}-\alpha^{2}\right)-\sqrt{1-2 \zeta^{2}-2 \alpha^{2}+\left(\zeta^{2}-\alpha^{2}\right)^{2}}}{\zeta \alpha} .
$$


For these values of $\theta_{-}$and relations (54), the energies $\mathcal{E}_{-}$coincide with (51).

The metric (53), (54) does not satisfy the Ricci flow equations with coordinates independent on RG time $t$ but there is a transformation of coordinates depending on $t$ which leads to the solution of these equations. This transformation from the metric (53) to (49) was constructed in [24].

For arbitrary group $G$ we can calculate the observable with the action (52) and $\alpha, \zeta$ defined by (54) where in relation (48) defining $\tau(t)$ we should take $q(t)=q_{G}(t)=\exp \left(\frac{h^{\vee}(G)\left(t-t_{0}\right)}{\left(p_{1}+p_{2}\right)}\right)$, where $h^{\vee}(G)$ is the dual Coxeter number. The factor $\Sigma(G)$ is simply related with the kernel $K_{G}(\omega)$ in the BA equations associated with the fundamental representation of $G$. $\Sigma(G)=$ $\frac{2}{K_{G}(0)}$. For $G=S U(n)$ this kernel is 4

$$
K_{S U(n)}=\frac{\sin \left(\frac{\pi \omega}{n}\right) \sin \left(\frac{(n-1) \pi \omega}{n}\right) \sin \left(\frac{\left(p_{1}+p_{2}\right) \omega}{n}\right)}{\sin (\pi \omega) \sin \left(\frac{p_{1} \omega}{n}\right) \sin \left(\frac{p_{2} \omega}{n}\right)}, \quad \Sigma(S U(n))=\frac{2 n p_{1} p_{2}}{(n-1)\left(p_{1}+p_{2}\right)}
$$

The GSE $\mathcal{E}(A)$ for the field $A$ coupled with $U(1)$ generated by one of generators $H_{i}$ from Cartan subalgebra for $S U(n)$, is

$$
\mathcal{E}(A)=-\frac{n p_{1} p_{2}}{2 \pi(n-1)\left(p_{1}+p_{2}\right)} A^{2} \tau(A) .
$$

Comparing the kernels (55) and (33), we see that $p_{1}=n-1, p_{2}=p$. In the scaling limit $p \gg 1$, we can solve the equation (48) by perturbation theory in $\frac{1}{p}$. In the first order we derive $\left.\left(\left(t_{0}-t\right)=\log \frac{A}{M}\right)\right)$

$$
\tau(A)=1-\frac{2(n-1)}{p} \frac{q^{2}}{1-q^{2}}+\cdots, \quad q(A)=q_{S U(n)}(A)=\exp \left(\frac{n \log \frac{A}{M}}{\left(p_{1}+p_{2}\right)}\right)
$$

It means that at the first order in $\frac{1}{p}$, the GSE $\mathcal{E}(A)$ defined by $(56)$ is

$$
\mathcal{E}(A)=-\frac{n}{2 \pi}\left(1-\frac{n-1}{p}\left(\frac{1+q^{2}}{1-q^{2}}\right)\right)+O\left(1 / p^{2}\right)
$$

in exact agreement with eq (40) derived in the BA approach.

We consider the function $\mathcal{E}_{-}(A)$ for $G=S U(2)$ (51) studied by the BA method in the previous section. Using relations (51,57) we obtain in the leading order in $\frac{1}{p}$

$$
\mathcal{E}_{-}(A)=-\frac{A^{2}}{\pi} p\left(\frac{1-q}{1+q}\right)+O(1)
$$

in exact agreement with (44) for $n=2$.

It is useful to consider the metric (49), which is the exact solution of Ricci equations in the limit $p_{1} \ll p_{2}=p$. In our case $p_{1}=1$. we derive from (49,57) that

$$
d s^{2}=4 p\left(\frac{1-q}{1+q}\right) \frac{d \theta^{2}+\sin ^{2} 2 \theta(d \beta)^{2}}{\left(1-\left(\frac{1-q}{1+q}\right)^{2} \cos ^{2} 2 \theta\right)}+O(1)
$$

\footnotetext{
${ }^{4}$ The scattering matrix of basic particles, i.e. particles with minimal mass in deformed principle chiral SM, $S(G)_{p_{1}, p_{2}}(\theta)$ is equal up to a simple CDD factor to the direct product of the well known $G_{\mathrm{q}}$ symmetric $S$-matricies $S(G)_{\mathrm{q}}$ in the fundamental (vector) representation of $G: S(G)_{p_{1}, p_{2}}=S(G)_{\mathrm{q}_{1}} \otimes S(G)_{\mathrm{q}_{2}}$ with $\mathrm{q}_{i}=$ $\exp \left(\mathrm{i} \frac{2 \pi}{p_{i}}\right)$.
} 
This metric is the sausage metric and describes the deformed $C P(1)$ model. The energy $\mathcal{E}_{-}(A)$ describes the degrees of freedom related with SM. The parameter $p_{1} \ll p_{2}=p$ does not appear in this limit. This parameter is related with additional degrees of freedom which are necessary for the quantum integrability of QFT.

The main term of the metric (60) (sausage metric) can be derived by the reduction procedure for coset $\frac{S U(2)}{U(1)}$. The deformed $C P(n-1)$ SM can be considered as SM on the coset space $\frac{S U(n)}{S U(n-1) U(1)}$. In general the action of SM on the coset space [20] can be written as

$$
\mathcal{A}_{c}=\frac{\delta^{\mu \nu}+\varepsilon^{\mu \nu}}{4 \pi} \Sigma \kappa \int d^{2} x\left(J_{a, \mu}\left(I+\mathrm{i} \kappa A\left(g_{c}\right) \mathcal{R} A\left(g_{c}\right)^{-1} P\right)_{a, b}^{-1} J_{a, \nu}\right)
$$

where $g_{c}$ is the coset element of the group from the numerator of coset, $P$ is the projector operator which annihilates the currents from denominator of coset and $\Sigma$ the numerical factor.

To simplify the expressions we consider here the case $n=3$, i.e. the deformed $C P(2) \mathrm{SM}$ (the same consideration can be done for any $n$, using eq(61) and results of appendix B). The matrix $g \subset S U(3)$ can be taken as (see appendix B)

$$
\begin{aligned}
g & =e^{\mathrm{i} \lambda_{3} \eta_{1}+\mathrm{i} \lambda_{8} \eta_{8}}\left(\begin{array}{ccc}
\cos \theta_{3} & 0 & e^{-\mathrm{i} \beta_{3}} \sin \theta_{3} \\
0 & 1 & 0 \\
-e^{\mathrm{i} \beta_{3}} \sin \theta_{3} & 0 & \cos \theta_{3}
\end{array}\right)\left(\begin{array}{ccc}
\cos \theta_{1} & e^{-\mathrm{i} \beta_{1}} \sin \theta_{1} & 0 \\
-e^{\mathrm{i} \beta_{1}} \sin \theta_{1} & \cos \theta_{1} & 0 \\
0 & 0 & 1
\end{array}\right) \\
& \times\left(\begin{array}{ccc}
1 & 0 & 0 \\
0 & \cos \theta_{2} & e^{-\mathrm{i} \beta_{2}} \sin \theta_{2} \\
0 & -e^{\mathrm{i} \beta_{2}} \sin \theta_{2} & \cos \theta_{2}
\end{array}\right)
\end{aligned}
$$

the coset element

$$
g_{c}=\left(\begin{array}{ccc}
e^{\mathrm{i} \beta_{1}} \cos \theta_{1} & e^{\mathrm{i} \beta_{2}} \cos \theta_{2} \sin \theta_{1} & \sin \theta_{1} \sin \theta_{2} \\
-\sin \theta_{1} & e^{\mathrm{i} \beta_{2}-\mathrm{i} \beta_{1}} \cos \theta_{2} \cos \theta_{1} & e^{-\mathrm{i} \beta_{1}} \sin \theta_{2} \cos \theta_{1} \\
0 & -\sin \theta_{2} & e^{-\mathrm{i} \beta_{2}} \cos \theta_{2}
\end{array}\right)
$$

In terms of these coordinates, we can calculate the metric $d s^{2}$ and the field $B$. We note that our theory for $n>2$ possesses only PT symmetry so that the $B$-field should appear. We introduce the notations

$$
\begin{aligned}
\mathrm{s}\left(\theta_{1}, \theta_{2}\right) & =1-2 \cos ^{2} \theta_{2} \sin ^{2} \theta_{1} \\
\mathrm{Z}\left(\theta_{1}, \theta_{2}, \kappa\right) & =\left(1-\kappa^{2} \cos ^{2}\left(2 \theta_{2}\right)\right)\left(1-\kappa^{2} \mathrm{~s}\left(\theta_{1}, \theta_{2}\right)\right)+\kappa^{2} \cos ^{4} \theta_{2} \sin ^{2}\left(2 \theta_{1}\right) \sin ^{2} \theta_{2} .
\end{aligned}
$$

Then we find that

$$
\begin{aligned}
d s^{2}= & \frac{\Sigma \kappa}{\mathrm{Z}\left(\theta_{1}, \theta_{2}, \kappa\right)}\left\{\cos ^{2} \theta_{2}\left(1-\kappa^{2} \cos ^{2}\left(2 \theta_{2}\right)\right)\left(d \theta_{1}\right)^{2}+\frac{\kappa^{2}}{2} \sin \left(4 \theta_{2}\right) \sin \left(2 \theta_{1}\right) d \theta_{1} d \theta_{2}\right. \\
& +\left(1-\kappa^{2}\left(\mathrm{~s}\left(\theta_{1}, \theta_{2}\right)-\cos ^{4} \theta_{2} \sin ^{2}\left(2 \theta_{1}\right) \sin ^{2} \theta_{2}\right)\right)\left(d \theta_{2}\right)^{2} \\
& +\cos ^{2} \theta_{2} \cos ^{2} \theta_{1}\left[\left(1-\cos ^{2} \theta_{2} \cos ^{2} \theta_{1}\right)-\kappa^{2}\left(\cos ^{2}\left(2 \theta_{2}\right) \sin ^{2} \theta_{1}+\cos ^{2} \theta_{1} \sin ^{2} \theta_{2}\right)\right]\left(d \beta_{1}\right)^{2} \\
& \left.-\frac{1}{2} \sin ^{2}\left(2 \theta_{2}\right) \cos ^{2} \theta_{1}\left(1-\kappa^{2} \mathrm{~s}\left(\theta_{1}, \theta_{2}\right)\right) d \beta_{1} d \beta_{2}+\frac{1}{4} \sin ^{2}\left(2 \theta_{2}\right)\left(1-\kappa^{2} \mathrm{~s}^{2}\left(\theta_{1}, \theta_{2}\right)\right)\left(d \beta_{2}\right)^{2}\right\}
\end{aligned}
$$


and the field

$$
\begin{aligned}
B= & \frac{\mathrm{i} \Sigma \kappa^{2}}{\mathrm{Z}\left(\theta_{1}, \theta_{2}, \kappa\right)}\left\{\sin \left(2 \theta_{1}\right) \cos ^{2} \theta_{2}\left(\left(1-\kappa^{2} \cos ^{2}\left(2 \theta_{2}\right)\right) \mathrm{s}\left(\theta_{1}, \theta_{2}\right)-\frac{\sin ^{2}\left(2 \theta_{2}\right)}{2} \cos ^{2} \theta_{1}\right) d \theta_{1} \wedge d \beta_{1}\right. \\
& +\frac{\sin \left(2 \theta_{1}\right)}{2} \cos ^{2} \theta_{2} \sin ^{2}\left(2 \theta_{2}\right) d \theta_{1} \wedge d \beta_{2}-\left(1-\kappa^{2} \mathrm{~s}\left(\theta_{1}, \theta_{2}\right)\right) \frac{\sin \left(4 \theta_{2}\right)}{2} \cos ^{2} \theta_{2} d \theta_{2} \wedge d \beta_{1} \\
& \left.+\frac{1}{2} \sin \left(4 \theta_{2}\right)\left(1-\kappa^{2} \mathrm{~s}^{2}\left(\theta_{1}, \theta_{2}\right)\right) d \theta_{2} \wedge d \beta_{2}\right\} .
\end{aligned}
$$

The metric (64) and the field $B$ (65) satisfy Ricci flow equations for $\Sigma=\frac{1}{\nu}$ and $\kappa(t)=$ $\tanh \left(6 \nu\left(t_{0}-t\right)\right)$. To show this, we use the conjecture, which is to be proven in the next paper, namely that Ricci flow equations are satisfied if they are satisfied for abelian $T$-dual SM. For $T$-dual SM with respect parameters $\beta_{1}, \beta_{2}$, the field $B$ disappears and the metric simplifies:

$$
\begin{aligned}
d s_{d u a l}^{2} & =\kappa(t)\left(\cos \theta_{1} d \theta_{2}+\frac{\mathrm{i} \tan \theta_{2}\left(\cos 2 \theta_{1}-\cot ^{2} \theta_{2}\right) d \beta_{1}-\mathrm{i} \cot \theta_{2} d \beta_{2}}{\cos \theta_{1}}\right)^{2} \\
& +\kappa(t)\left(d \theta_{1}-2 \mathrm{i} \cot 2 \theta_{1} d \beta_{2}\right)^{2}+\frac{\left(\left(\frac{d \beta_{1}}{\cos \theta_{2}}+\cos \theta_{2} d \beta_{2}\right)^{2}+\frac{\sin ^{2} \theta_{2}}{\sin ^{2} \theta_{1}} d \beta_{2}^{2}\right)}{\kappa(t) \sin ^{2} \theta_{2} \cos ^{2} \theta_{2}}
\end{aligned}
$$

It is easy to check that Ricci flow equations

$$
\frac{d}{d t} g_{i j}=-R_{i j}+2 \nabla_{i} \nabla_{j} \Phi
$$

are satisfied with dilaton field $\Phi=\log \left(\cos \theta_{1} \sin 2 \theta_{1} \sin 2 \theta_{2}\right)+4 \mathrm{i}\left(\beta_{1}+2 \beta_{2}\right)$ and

$$
\kappa(t)=\frac{\tanh \left(6 \nu\left(t_{0}-t\right)\right)}{\nu}
$$

To compare the observables calculated from the SM representation with that's derived from scattering theory data by the BA method, we introduce the fields related with two $U(1)$ symmetries of SM (64), (65). Namely $\partial_{0} \beta_{i} \rightarrow\left(\partial_{0}+\mathrm{i} A_{i}\right) \beta_{i}$. The configuration considered by the BA method corresponds $A_{1}=-A_{2}=A$. In field $A,\left(t_{0}-t\right)=\log \frac{A}{M}$. It is easy to find that the minimum of the SM action corresponds to $\theta_{1}=0, \theta_{2}=\frac{\pi}{4}$. The minimum of the action defines the ground state energy $\mathcal{E}_{-}(A)$ for the deformed $\mathrm{SM}$ and is given by

$$
\mathcal{E}_{-}(A)=-\frac{A^{2}}{4 \pi \nu} \tanh \left(6 \nu \log \frac{A}{M}\right) .
$$

For deformed $C P(1)$ SM we had from eq(159) that

$$
\mathcal{E}_{-}(A)=-\frac{A^{2}}{\pi} p \tanh \left(\frac{1}{p} \log \frac{A}{M}\right)=-\frac{A^{2}}{4 \pi \nu} \tanh \left(4 \nu \log \frac{A}{M}\right) .
$$

Comparing these equations it is natural to assume that $\nu=\frac{1}{4 p}$ and for the deformed $C P(n-1)$ SMs one obtains

$$
\mathcal{E}_{-}(A)=-\frac{A^{2}}{\pi} p \tanh \left(\frac{n}{2 p} \log \frac{A}{M}\right)
$$

in exact agreement with the BA calculations (44). The parameter $\Sigma=\frac{1}{\nu}=4 p, p=1+b^{2}(35)$. It provides a relation between the parameters of QFTs (15), (25) and sigma models. 


\section{Conformal limit of metric, semiclassical reflection am- plitudes and UV asymptotic of central charge}

In the previous section we conjectured a duality between the deformed $C P(n-1) \mathrm{SM}$ with fermion and QFT described by the action (15),(24). Here we consider a duality between the CFT with action (6) and conformal limit of the metric of deformed $C P(n-1)$ SM. The free term in action $\mathcal{A}_{C F T}$ corresponds to fermion. For simplification we restrict ourselves to the case $n=3$. The case $n=2$, corresponding to the duality between the Sine-Liouville CFT and Witten's $2 d$ black hole SM, was considered in [23].

To derive the conformal limit of the metric $d s^{2}$ and the $B$-field, we use the method of contraction [22]. We denote as $u$ the parameter

$$
u=\frac{1}{p}\left(t_{0}-t\right)=\frac{1}{1+b^{2}}\left(t_{0}-t\right) .
$$

In UV limit $u \rightarrow \infty, \kappa(u)=\tanh \frac{3}{2} u \rightarrow 1$. We introduce the parameter $\delta / 2=1-\tanh u \rightarrow 0$ and make the rescaling of variables $\vartheta_{1} \rightarrow \sqrt{\delta} / 2 \vartheta_{1}, \vartheta_{2} \rightarrow \sqrt{\delta} / 2 \vartheta_{2}$ with $\delta \rightarrow 0$. As a result, the conformal limit of the metric $d s^{2}$ can be written as $\left(\frac{1}{\nu}=4 p\right)$

$$
\begin{aligned}
d s_{C}^{2} & =p \frac{\left(1+\theta_{2}^{2}\right)\left(d \theta_{1}\right)^{2}+\left(1+\theta_{1}^{2}\right)\left(d \theta_{2}\right)^{2}+\theta_{1} \theta_{2} d \theta_{1} d \theta_{2}}{\left(1+\theta_{1}^{2}+\theta_{2}^{2}+\frac{3}{4} \theta_{1}^{2} \theta_{2}^{2}\right)} \\
& +p \frac{\left(\theta_{1}^{2}+\theta_{2}^{2}+\theta_{1}^{2} \theta_{2}^{2}\right)\left(d \beta_{1}\right)^{2}+\theta_{2}^{2}\left(1+\theta_{1}^{2}\right)\left(d \beta_{2}\right)^{2}-\left(2+\theta_{1}^{2}\right) \theta_{2}^{2} d \beta_{1} d \beta_{2}}{\left(1+\theta_{1}^{2}+\theta_{2}^{2}+\frac{3}{4} \theta_{1}^{2} \theta_{2}^{2}\right)}
\end{aligned}
$$

and the field $B$

$$
\begin{gathered}
B_{C}=\mathrm{i} p \frac{\theta_{1}\left(1+\frac{\theta_{2}^{2}}{2}\right) d \theta_{1} \wedge d \beta_{1}-\theta_{2}\left(1+\frac{\theta_{1}^{2}}{2}\right) d \theta_{2} \wedge d \beta_{1}}{\left(1+\theta_{1}^{2}+\theta_{2}^{2}+\frac{3}{4} \theta_{1}^{2} \theta_{2}^{2}\right)} \\
-\mathrm{i} p \frac{\frac{1}{2} \theta_{1} \theta_{2}^{2} d \theta_{1} \wedge d \beta_{2}+\theta_{2}\left(1+\theta_{1}^{2}\right) d \theta_{2} \wedge d \beta_{2}}{\left(1+\theta_{1}^{2}+\theta_{2}^{2}+\frac{3}{4} \theta_{1}^{2} \theta_{2}^{2}\right)}
\end{gathered}
$$

The action of SM with the metric (72) and the $B$-field (73) can be written in terms of two complex scalar fields $\omega_{1}(x)=\theta_{1} e^{\mathrm{i} \beta_{1}}, \omega_{1}(x)=\theta_{2} e^{\mathrm{i} \beta_{2}}$. It describes CFT corresponding coset $\frac{S L(3)_{K}}{S L(2)_{K} U(1)}$ with $K=3+b^{2}$. This CFT can be used for construction of string theory moving on such coset manifold.

To identify our conformal SM with CFT (6), we show that the reflection amplitudes derived in the minisuperspace approach in our conformal SM coincide with the semiclassical limit of the reflection amplitudes (13). Here we consider our SM on a circle of length $R$. We have a scale and $\left(t_{0}-t\right)=\log \left(\frac{1}{M R}\right)$. In the UV scaling regime $\log \left(\frac{1}{M R}\right) \rightarrow \infty, \frac{1}{p} \rightarrow 0$, with $u$ defined in (71), our approximation is exact up to $O\left(\frac{1}{p} \log p\right)$. In this approximation we can use minisuperspace approach to calculate ground state energy $E_{0}(R)$, effective central charge $E_{0}(R)=-\frac{\pi c_{\mathrm{eff}}(R)}{R}$ and energies $E_{i}(R)$ of exited states. Let $c_{\mathrm{eff}}(R)=c_{U V}-e_{0}(R), E_{i}(R)=\frac{\pi e_{i}}{R}$. Then the minisuperspace equation for the spectrum and eigenfunctions has the form [22]

$$
\left(-\nabla^{2}+\frac{1}{4} \mathcal{R}+\frac{1}{12} \mathcal{H}^{2}\right) \Psi_{i}=\frac{e_{i}}{6} \Psi_{i}
$$


where $\mathcal{R}$ is the curvature and 3 -form $\mathcal{H}$ is defined in terms of two form $B$ as $\mathcal{H}=d B$.

The operator in the l.h.s. of equation (74) can be written as $p \mathrm{H}$, where the operator $\mathrm{H}$ depends only on $u$. It means that $\mathrm{e}_{i}=\frac{e_{i}}{p}$ are functions only on $u$. A solution of this equation with the metric and the $B$-field for the deformed $C P(n-1) \mathrm{SM}$ is accessible only numerically. Even for $n=2$ where $B=0$, eq. (74) reduces to Lamé equation which can be solved analytically only in the limits of small and large values of the scaling variable $u$. For $u<<1$ the metric and $\mathcal{R}$ reduce to those of $C P(n-1)$ and the spectrum is trivial. The corrections in $u$ can be derived by the standard perturbation theory. In particular,

$$
\mathrm{e}_{0}(u)=p\left(c_{U V}-c_{\mathrm{eff}}(R)\right)=\frac{3 n}{2 u}\left(1+O\left(u^{4}\right)\right) .
$$

For $u>>1$ the calculations are more involved. The starting point is the conformal limit of the action of SM. Here we consider the case $n=3$, where this action is given by (72), (73)).

It is convenient to parametrize the spectrum of the eq (174) as $\mathrm{e}_{0}=12 P^{2}=12\left(P_{1}^{2}+P_{2}^{2}\right)$. The functions $\Psi_{i}(\operatorname{det} G)^{1 / 4}$, which are integrated with trivial measure, will be denoted as $\Psi_{P}$.

The Weyl group $\mathbf{w}_{3}$ of the Lie algebra of $s l(3)$ contains 6 elements and acts to the parameters $\left(P_{1}, P_{2}\right)$ as rotations in this plane by $\frac{2 \pi l}{3}$ and reflection $P \rightarrow-P$. We denote $P_{s}=\widehat{s} P$. For calculation of the effective central charge, we can consider functions $\Psi_{P}$, independent of $\beta_{1}, \beta_{2}$. To simplify the notations and equations, we consider here the isotopic symmetric sector where $\Psi_{P}=\Psi_{P}\left(\theta_{1}, \theta_{2}\right)$. It is convenient to make the substitution $\theta_{i}=\sqrt{Y_{i}}$ and $\Psi_{P}=F\left(Y_{1}, Y_{2}\right)$, the we obtain from eq(74) that

$$
\begin{aligned}
0 & =\left(Y_{1} F_{Y_{1}}+Y_{2} F_{Y_{2}}+Y_{1}\left(1+Y_{1}\right) F_{Y_{1}, Y_{1}}+Y_{2}\left(1+Y_{2}\right) F_{Y_{2}, Y_{2}}+Y_{1} Y_{2} F_{Y_{1}, Y_{2}}\right) \\
& +\frac{1}{8 Y_{1} Y_{2}}\left(2 Y_{1}+2 Y_{1}+4\left(P_{1}^{2}+P_{2}^{2}\right)\right) F .
\end{aligned}
$$

This equation can be soled by the Mellin transform method.

$$
F\left(Y_{1}, Y_{2}\right)=\int_{C} Y_{1}^{\mathrm{i} Q_{1}} Y_{2}^{\mathrm{i} Q_{2}} \widetilde{F}\left(Q_{1}, Q_{2}\right) d Q_{1} d Q_{2}
$$

where the contour $C$ includes the poles of $\widetilde{F}\left(Q_{1}, Q_{2}\right)$ on the real axe $\widetilde{F}\left(Q_{1}, Q_{2}\right)$. The equation (76) $)$ can be reduced to a functional equation to $\widetilde{F}\left(Q_{1}, Q_{2}\right)$

$$
\begin{aligned}
\left(\frac{P^{2}}{2}-Q_{1}^{2}-Q_{2}^{2}+Q_{1} Q_{2}\right) \widetilde{F}\left(Q_{1}, Q_{2}\right) & =\left(\frac{1}{2}-\mathrm{i} Q_{1}\right)^{2} \widetilde{F}\left(Q_{1}-\mathrm{i}, Q_{2}\right) \\
& +\left(\frac{1}{2}-\mathrm{i} Q_{2}\right)^{2} \widetilde{F}\left(Q_{2}, Q_{2}-\mathrm{i}\right) .
\end{aligned}
$$

A solution to this equation can be found in a similar way as it was done in [47] for sl (3) Toda equation. Namely, let $h_{i}$ be the weights of the fundamental representation. Then

$$
\widetilde{F}\left(Q_{1}, Q_{2}\right)=\frac{\Gamma^{2}\left(\frac{1}{2}-\mathrm{i} Q_{1}\right) \Gamma^{2}\left(\frac{1}{2}-\mathrm{i} Q_{2}\right) \prod_{j=1}^{3} \Gamma\left(\mathrm{i} Q_{1}+\mathrm{i} h_{j} \cdot P\right) \Gamma\left(\mathrm{i} Q_{2}-\mathrm{i} h_{j} \cdot P\right)}{\Gamma\left(\mathrm{i} Q_{1}+\mathrm{i} Q_{2}\right)}
$$

This function is Weyl invariant. The contributions to the asymptotics of the function $F\left(Y_{1}, Y_{2}\right)$ give the poles at $Q_{1}=-h_{j} \cdot P, Q_{2}=h_{m} \cdot P$ with $m \neq j$. If we take into account that the 
roots of $\operatorname{sl}(n)$ are $h_{j}-h_{m}, m \neq j$, and denote $Y_{1}=e^{e_{1} \cdot X}, Y_{2}=e^{e_{2} \cdot X}$, we find that in the Weyl chamber the function $F\left(Y_{1}, Y_{2}\right)$ has the asymptotics

$$
F\left(e^{e_{1} \cdot X}, e^{e_{2} \cdot X}\right)=\sum_{w_{3}} A\left(P_{s}\right) e^{\mathrm{i} P_{s} \cdot X}
$$

where

$$
A(P)=\Gamma^{2}\left(\frac{1}{2}+\mathrm{i} h_{1} \cdot P\right) \Gamma^{2}\left(\frac{1}{2}-\mathrm{i} h_{3} \cdot P\right) \prod_{\alpha>0} \Gamma\left(-e_{\alpha} \cdot P\right) .
$$

We see that the function $A(P)$ coincides with scaling, or semiclassical limit ( i.e. $b>>1$, $\mathbf{a} b=\mathrm{i} P$ is fixed) of quantum amplitude $\mathbf{A}(\mathbf{a}, 0,0)$ (12). It means that the conformal limit of our SM is dual to CFT (6) .

Using the amplitudes $\mathbf{A}(\mathbf{a}, \mathbf{0}, 0)(12)$ we can write the equations for quantization of $P$. This procedure is explained in details in [45]. In semiclassical limit they have the form

$$
2 u n P+\sum_{\alpha>0} e_{\alpha} f_{1}\left(e_{\alpha} \cdot P\right)+n \sum_{j=1}^{n} h_{j} f_{2}\left(h_{j} \cdot P\right)=2 \pi\left(\rho+\Omega_{n}\right),
$$

where $f_{1}(z)=\frac{1}{\mathrm{i}} \log \left(\frac{\Gamma(1+\mathrm{i} z)}{\Gamma(1-\mathrm{i} z)}\right), f_{2}(z)=\frac{1}{\mathrm{i}} \log \left(\frac{\Gamma\left(\frac{1}{2}-\mathrm{i} z\right)}{\Gamma\left(\frac{1}{2}+\mathrm{i} z\right)}\right)$, and $\Omega_{n}$ is the highest weight of $\operatorname{su}(n)$.

In the deep UV region $u>>1$, we can expand this equation up to the first order in $P$. We have $\Sigma_{\alpha>0}\left(e_{\alpha}\right)_{s}\left(e_{\alpha}\right)_{r}=n \delta_{s, r}, \Sigma_{j}^{n}\left(h_{j}\right)_{s}\left(h_{j}\right)_{r}=\delta_{s, r}$ and $\frac{\Gamma^{\prime}(1)}{\Gamma(1)}-\frac{\Gamma^{\prime}\left(\frac{1}{2}\right)}{\Gamma\left(\frac{1}{2}\right)}=\log 4$. Then we derive the semiclassical quantization of $P$,

$$
P=\frac{\pi\left(\rho+\Omega_{n}\right)}{n(u+n \log 4)}
$$

For $\mathrm{e}_{0}=12 P^{2}=p\left(c_{U V}-c_{\text {eff }}(R)\right)$, we obtain that for $u>>1$ one has

$$
\mathrm{e}_{0}(u)=\frac{12 \pi^{2} \rho^{2}}{n^{2}(u+n \log 4)^{2}}+O\left(1 / u^{5}\right)=\frac{\pi^{2}\left(n^{2}-1\right)}{n(u+n \log 4)^{2}}+O\left(1 / u^{5}\right) .
$$

It implies that $\mathrm{e}_{0}(u)=\frac{3 n}{2 u}\left(1+O\left(u^{4}\right)\right)$ for $u<<1$ and $\mathrm{e}_{0}(u)=\frac{\pi^{2}\left(n^{2}-1\right)}{n(u+n \log 4)^{2}}+O\left(1 / u^{5}\right)$ for $u>>1$. Between these values, $\mathrm{e}_{0}(u)$ is monotonically decreasing (due to $c$-theorem [29]) function of $u$. We note that $p=1+b^{2} \approx b^{2}>>1$. It means that

$$
c_{\mathrm{eff}}(R)-c_{U V} \approx-\frac{3 n}{2 \log \left(\frac{1}{M R}\right)} \quad ; \quad c_{\mathrm{eff}}(R)-c_{U V} \approx-\frac{\pi^{2}\left(n^{2}-1\right) b^{2}}{n\left(\log \left(\frac{1}{M R}\right)+b^{2} n \log 4\right)^{2}}
$$

for $b^{2}>>\log \left(\frac{1}{M R}\right)$ and $b^{2}<<\log \left(\frac{1}{M R}\right)$.

The exited levels $\mathrm{e}_{i}(u)-\mathrm{e}_{0}(u)$ in the "isotopic symmetric" sector depend on $n-1$ quantum numbers and flow from $\lambda_{i} / u$, where $\lambda_{i}$ are the eigenvalues of Laplacian on $C P(n-1)$ for small $u$, to $\frac{12 \pi^{2}\left(\Omega_{n}^{2}+2 \rho \cdot \Omega_{n}\right)}{n^{2}(u+n \log 4)^{2}}$ for large $u$.

\section{Integrable perturbation $\mu_{1} e^{b\left(e_{0} \cdot \varphi\right)}$}

In this Section we briefly consider the second integrable perturbation of CFT (6) $)$. With this perturbation the action will have the following form

$$
\mathcal{A}_{s l(n)}^{(2)}=\mathcal{A}_{C}+\mu_{1} \int d^{2} x e^{b\left(e_{0} \cdot \varphi\right)},
$$


where $e_{0}$ is affine root of $\operatorname{sl}(n)$.

We can use again the Coleman-Mandelstam $2 d$ correspondence between fermion and bosons (22). We introduce the fields

$$
\xi_{i}=a\left(\eta_{i} \cdot \vartheta\right)+\vartheta_{0} \frac{\sqrt{n+b^{2}}}{\sqrt{n(n-1}} ; \quad\left(\eta_{i} \cdot \eta_{j}\right)=1-\frac{\delta_{i, j}}{n-1} ; \quad i=1, . . n-1
$$

and projectors $\gamma_{+}, \gamma_{-}: \gamma_{ \pm}=\frac{1}{2}\left(1 \pm \gamma_{5}\right)$. Then the action (82) after application of ColemanMandelstam rules can be rewritten in the form suitable for perturbation theory for small $b$.

$$
\mathcal{A}_{s l(n)}^{(2)}=\int d^{2} x\left(L_{F}^{(2)}+L_{B}^{(2)}+L_{F B}^{(2)}\right)
$$

where $L_{F}^{(2)}$ and $L_{F B}^{(2)}$ can be derived from $L_{F}$ and $L_{F B}$ in (25) by taking the sums from 1 to $n-1$ and substitution $g_{1} \rightarrow g_{1}^{(2)}=-\frac{b^{2}\left(n-1+b^{2}\right)}{\left(1+b^{2}\right)\left(n+b^{2}\right)}, g_{2} \rightarrow g_{2}^{(2)}=\frac{b^{2}}{\left(n+b^{2}\right)\left(1+b^{2}\right)}$.

This QFT has a broken $\mathbf{P}$ symmetry $(n>2)$ but possesses $\mathbf{P T}$ and $\mathbf{C}$ symmetries. For small $b$ it has $2(n-1)$ particles $\psi_{i}, \psi_{i}^{*}$ with mass $m$, and $n-1$ scalar particles $\varphi_{i}$ which can be considered as bound states of Fermi particles with masses $m_{j}=2 m \sin \left(\frac{\pi j}{n}\right)+O\left(b^{2}\right)$. For finite $b$ they disappear from the spectrum.

The scattering theory now is described by the eqs 26 where in $S$ - matrix $S^{(2)}(\theta)$ the indices $i \rightarrow \psi_{i}, \bar{i} \rightarrow \psi_{i}^{*}$ now take values $1, . ., n-1$. In the Eqs.(26) we also take the parameters $n \rightarrow \mathrm{n}=\mathrm{n}\left(\mathrm{b}^{2}\right)$ and $\lambda=\lambda\left(b^{2}\right)$. The unitarizing factor $F(\theta)$ now becomes

$$
F_{\mathrm{n}, \lambda}^{(2)}(\theta)=\exp \left(\mathrm{i} \int_{-\infty}^{\infty} \frac{d \omega}{\omega} \frac{\sinh \left(\frac{\pi(\mathrm{n}-1) \omega}{\mathrm{n}}\right) \sinh \left(\frac{\pi(p-1) \omega}{\mathrm{n}}\right)}{\sinh (\pi \omega) \sinh \left(\frac{\pi p \omega}{\mathrm{n}}\right)} \sin (\omega \theta)\right) ; \quad \lambda=\frac{1}{p} .
$$

The dependence of the parameters $\mathrm{n}\left(\mathrm{b}^{2}\right)$ and $\lambda\left(b^{2}\right)$ can be derived from BA equations for GSE in external fields $A_{i}$ coupled with $n-1$ conserved $U(1)$ charges $Q_{i}=\int \psi_{i}^{*} \psi d^{2} x$. Following the lines of Section 5, we find that

$$
\frac{1}{\lambda}=p=\left(1+b^{2}\right) ; \quad \mathrm{n}=\left(n+b^{2}\right) .
$$

At the limit $b^{2} \rightarrow \infty$,the scattering matrix $S^{(2)}(\theta)$ tends to the identity matrix and can be expanded in $1 / b^{2}=\mathrm{b}^{2}$. It is important to note that the function $F_{\mathrm{n}, \lambda}^{(2)}(\theta)$ (84) at small $b^{2}$ is $1+O\left(b^{2}\right)$ and amplitudes $\psi_{i} \psi_{i} \rightarrow \psi_{i} \psi_{i}$ equal to $-F_{\mathrm{n}, \lambda}^{(2)}(\theta) \rightarrow-1$ at $\theta \rightarrow 0$, what is characteristic property for fermionic particles, for $b^{2} \rightarrow \infty$ we get that this amplitude $-F_{\mathrm{n}, \lambda}^{(2)}(\theta) \rightarrow 1+O\left(\mathrm{~b}^{2}\right)$, as it happens for bosonic particles. This phenomenon (fermion-boson duality) is easy to see in the case $n=2$ where we have only one charged particle $\psi, \psi^{*}$, and hence, $S$-matrix is a pure phase. The action (83) is described by $L_{s l(2)}^{(2)}$, which is:

$$
\left.\frac{1}{8 \pi}\left(\partial_{\mu} \varphi\right)^{2}+2\left(\mathrm{i} \bar{\psi} \gamma_{\mu} \partial_{\mu} \psi+m \overline{\psi \psi} e^{b \varphi / \sqrt{2}}-\frac{b^{2}}{2+b^{2}}\left(\bar{\psi} \gamma_{\mu} \psi\right)^{2}\right)+m^{2} \cosh (\sqrt{2} b \varphi)\right)
$$

The scattering matrix $S_{\psi \psi}=-F_{\mathrm{n}, \lambda}^{(2)}(\theta)$ for $n=2$ can be calculated and is given by

$$
S_{\psi \psi}(\theta)=-\frac{\cosh (\theta / 2+\mathrm{i} \Delta / 2)}{\cosh (\theta / 2-\mathrm{i} \Delta / 2)}, \quad S_{\psi \psi^{*}}=S_{\psi \psi}(\mathrm{i} \pi-\theta), \quad \Delta=\frac{\pi b^{2}}{1+b^{2}} .
$$


For $b>>1$ it can be expanded as a regular series in $1 / b^{2}=\mathrm{b}^{2}$. In this region our QFT can be described in terms of one complex scalar field $\omega(x)$ by the action [18]

$$
\mathcal{A}_{s l(2)}^{(2)}=\int d^{2} x \frac{1}{4 \pi}\left(\frac{\partial_{\mu} \omega \partial_{\mu} \omega^{*}}{1+\mathrm{b}^{2} \omega \omega^{*}}+m^{2} \omega \omega^{*}\right) .
$$

We see that charged particles being fermions in the weak coupling region, become bosons in the strong coupling region.

The SM part of the action (87) coincides with the dual action for CFT (66) $(n=2)$, or with the conformal limit of metric of deformed $C P(1)$ model. The potential part $m^{2} \omega \omega^{*}$ defines the tachyon. In general case (arbitrary $n$ ) bosonic QFTs dual to (83) for small b have the classical limit. They define classical integrable models, which we call conventually "non-abelian $\operatorname{sl}(n)$ affine Toda theories".

The SM part of these QFTs is described by the conformal limit of the metric $G_{i j}^{(c)}$ and the $B^{(c)}$-field of deformed $C P(n-1)$ model. Together with potential (tachyon) the dual action can be expressed in terms of $n-1$ complex fields $\omega_{i}(x)$.

$$
\mathcal{A}_{s l(n)}^{(2)}=\int \frac{d^{2} x}{4 \pi}\left(G_{i j}^{(c)}\left(\mathrm{b} \omega_{i}, \mathrm{~b} \omega_{i}^{*}\right)\left(\partial_{\mu} \omega_{i} \partial_{\mu} \omega_{j}^{*}+\varepsilon_{\alpha \beta} B_{i j}^{(c)} \partial_{\alpha} \omega_{i} \partial_{\beta} \omega_{j}^{*}\right)+\frac{m^{2}}{\mathrm{~b}^{2}} \mathrm{\top}\right),
$$

where $\mathrm{T}=\frac{m^{2}}{\mathrm{~b}^{2}} \mathrm{~T}\left(\mathrm{~b} \omega_{i}, \mathrm{~b} \omega_{i}^{*}\right)$ denotes the tachyon. Contrary to the scattering theory and observables, the functions $G_{i j}\left(\omega_{i}, \omega_{i}^{*}\right), B_{i j}\left(\omega_{i}, \omega_{i}^{*}\right)$ and $\mathrm{T}\left(\omega_{i}, \omega_{i}^{*}\right)$ can depend on the choice of coordinates (see appendix $(\mathrm{B})$. We hope to return to this problem in a separate publication.

In the end of this section we make some important remark, not related to the duality. In the action $\mathcal{A}_{s l(n)}^{(2)}$, which has a classical limit, we can take parameter b purely imaginary: $\mathrm{b} \rightarrow \mathrm{i} \gamma$. The classical theory will be integrable and well-defined. However, due to the singularities of the metric and the $B$-field, the quantization of this theory will be more subtle. For example, the classical theory (87) after $\mathrm{b} \rightarrow \mathrm{i} \gamma$ is known as complex Sine-Gorgon or Lund-Regge [36] model. In quantum case we can perform in $S$-matrix (86) the transformations $b \rightarrow \frac{1}{\mathrm{~b}}, \mathrm{~b} \rightarrow \mathrm{i} \gamma$, and denote $S_{\psi \psi}(\theta) \rightarrow S_{\omega \omega}(\theta)$.

$$
S_{\omega \omega}(\theta)=-\frac{\sinh \left(\theta / 2+\mathrm{i} \Delta_{1} / 2\right)}{\sinh \left(\theta / 2-\mathrm{i} \Delta_{1} / 2\right)} ; \quad S_{\omega \omega^{*}}(\theta)=S_{\omega \omega}(\mathrm{i} \pi-\theta), \quad \Delta_{1}=\frac{\pi \gamma^{2}}{1-\gamma^{2}} .
$$

These amplitudes are in exact agreement with perturbation theory in $\gamma$. However, the amplitude $S_{\omega \omega}(\theta)$ possesses the pole in the physical strip, corresponding to a bound state of two particles $\omega(\theta)$. This particle has $U(1)$ charge equal twice the charge of $\omega(\theta)$. For general values of $\gamma$ the $S$-matrix bootstrap is not closed and the charge of particles is not limited. This contradiction disappears only for special values of $\gamma$, namely when

$$
\Delta_{1}=\frac{\pi \gamma^{2}}{1-\gamma^{2}}=\frac{\pi}{k}, \quad \gamma^{2}=\frac{1}{k+n}, \quad k=0,2,3 .
$$

It means that we have a well-defined QFT only for special values of $\gamma$ (quantization of coupling constant). The similar phenomenon has place for all values of $n$. The analysis of scattering theory shows that theory is well-defined only for $\gamma^{2}=\frac{1}{k+n}$ with positive integer $k .5$ The

\footnotetext{
${ }^{5}$ The similar phenomenon appears in the non-unitarian imaginary Toda QFT which is described by the Lagrangian $L_{B}$ (25) with purely imaginary $b=\mathrm{ib}$. In this QFT the scattering bootstrap is closed only for $\mathrm{b}^{2}=\frac{p}{p+1}$, where $p$ is integer and $p>h$. For these values of the coupling constant the imaginary Toda theory, after the quantum group restriction, becomes a unitary QFT.
} 
condition

$$
\frac{1}{\gamma^{2}}=-b^{2}=k+n
$$

will be considered in the next section.

\section{Rational CFTs, their perturbations and sigma models}

In this Section we discuss some important points (in particular, another integrable deformations of SMs) which were not considered in the main body of this paper.

1. In section 6 we discussed the SMs and Ricci flows related with massive QFTs. However there are massless Ricci flows associated with SMs with singular metrics which for special values of the parameter $b$ describe the flows from CFTs with continuous spectrum in the UV regime to rational unitarian CFTs in IR regime (See [46],[23], [22]). For deformed $C P(n-1)$ the SM representation dual to CFT (6) is described by the action (88) with $b^{2}>0$ and $T=0$. The Ricci flow with singular metric relates CFT (88) with $b^{2}=n+k$ and rational CFT with $-b^{2}=n+k$ (90).

The central charge of CFT (66) with $b^{2}=-(n+k), a^{2}=-(n+k-1)$ is (17)

$$
\mathrm{c}_{n, k}=(n-1)\left(2-\frac{(n+1) n}{k+n}+\frac{n(n-2)}{k+n-1}\right)
$$

here we ignore for the moment 1 coming from free field $\vartheta_{n}$. This field will appear later.

The central charge (91) corresponds to the coset $\frac{S U(n)_{k}}{S U(n-1)_{k} U(1)}$. These minimal models $\mathcal{M}(n, k)$ are characterized by strongly degenerate representations of $W$-algebras $\mathcal{W}(n, k)$ which commute with all screening charges with densities $\left\{V_{1}, V_{-2}\right\}, . .,\left\{V_{n-1}, V_{-n}\right\}$ (4) and can be represented by $2(n-1)$ free fields $(n-1)$ dimensional vector $\varphi,(n-2)$ dimensional $\vartheta$ and field $\vartheta_{0}$. The primary fields in $\mathcal{M}(n, k)$ can be represented as

$$
\Phi\left(\boldsymbol{\Omega}_{n}, \boldsymbol{\Omega}_{n-1}, s\right)=\exp \left(\mathrm{i} \frac{\boldsymbol{\Omega}_{n} \cdot \varphi}{\sqrt{n+k}}-\frac{\boldsymbol{\Omega}_{n-1} \cdot \vartheta}{\sqrt{n+k-1}}-\frac{\vartheta_{0} s}{\sqrt{k n(n-1)}}\right)
$$

where $\Omega_{n}$ is the highest weight vector of $s u(n)$ and $\pm s$ is integer with the condition

$$
k \geq-\boldsymbol{\Omega}_{n} \cdot\left(\mathbf{e}_{0}\right)_{n} \geq-\boldsymbol{\Omega}_{n-1} \cdot\left(\mathbf{e}_{0}\right)_{n-1} \geq|s|
$$

The dimensions of these fields

$$
\Delta\left(\boldsymbol{\Omega}_{n}, \boldsymbol{\Omega}_{n-1}, s\right)=\frac{1}{2}\left(\frac{\boldsymbol{\Omega}_{n} \cdot\left(\boldsymbol{\Omega}_{n}+2 \rho_{n}\right)}{n+k}-\frac{\boldsymbol{\Omega}_{n-1} \cdot\left(\boldsymbol{\Omega}_{n-1}+2 \rho_{n-1}\right)}{n+k-1}-\frac{s^{2}}{k n(n-1)}\right) .
$$

In particular, the receiving field of Ricci flow is the descendent field $\boldsymbol{\Phi}_{R}=\left(\mathcal{W}_{3}\right)_{-1} \mathbf{\Phi}(\mathbf{a d}, \mathbf{0}, 0)$ with dimension $1+\frac{n}{k+n}$.

For many applications it is useful to use the level-rank duality [21] which relates $\frac{S U(n)_{k}}{S U(n-1)_{k} U(1)}$ and $\frac{S U(k)_{n-1} S U(k)_{1}}{S U(k)_{n}}$ minimal models. The minimal models $\mathcal{M}(k, n)=\frac{S U(k)_{n-1} S U(k)_{1}}{S U(k)_{n}}$ are well known. They possess $W_{k}$-symmetry associated with the Lie algebra $s u(k)$ and central charge $c_{k, n}=(k-1)\left(1-\frac{k(k+1)}{(k+n-1)(k+n)}\right)$. It is easy to see that $c_{k, n}=c_{n, k}$. The space of primary fields 
$\Phi_{k}\left(\Omega, \Omega^{\prime}\right) \in \mathcal{M}(k, n)$ is specified by two highest weight vectors of $s u(k)$ : $\Omega$ and $\Omega^{\prime}$ which satisfy the conditions: $-\Omega \cdot e_{0} \leq n$, and $-\Omega^{\prime} \cdot e_{0} \leq n-1$ and have the dimensions

$$
\Delta\left(\Omega, \Omega^{\prime}\right)=\frac{1}{2}\left(\frac{a}{b} \Omega-\frac{b}{a} \Omega^{\prime}\right)\left(\frac{a}{b} \Omega-\frac{b}{a} \Omega^{\prime}+\frac{2}{a b} \rho_{k}\right),
$$

where $b=\mathrm{i} \sqrt{k+n}, a=\mathrm{i} \sqrt{k+n-1}$. In particular the receiving field of Ricci flow is the descendent field $\Phi_{R}=\left(W_{3}\right)_{-1} \Phi_{k}(a d, 0)$ with dimension $1+\frac{n}{k+n}$.

All currents of $W_{k}$-algebra (which we call for fixed $n$ as $W_{k}(n)$ ) can be represented by $k-1$ free fields $\chi$ and commute with screenings generated by fields $e^{\mathrm{i} \frac{a}{b} e_{j} \cdot \chi}, e^{-\mathrm{i} \frac{b}{a} e_{j} \cdot \chi}$. The fields $\Phi_{k}\left(\Omega, \Omega^{\prime}\right)$ can be represented as

$$
\Phi_{k}\left(\Omega, \Omega^{\prime}\right)=\exp \left(\mathrm{i} \frac{a}{b} \Omega \cdot \chi-\mathrm{i} \frac{b}{a} \Omega^{\prime} \cdot \chi\right)
$$

It means that we have two different representations for the same CFT $\mathcal{M}(k, n)=\mathcal{M}(n, k)$. We call $W_{k}(n)$ as "vertical" $W$-algebra and $\mathcal{W}(n, k)$ as "horizontal" $W$-algebra of CFT $\mathcal{M}(k, n)$. The existence of two different representations for the same CFT and as a result two different integral representations for the correlation functions will be studied in a separate publication. Here we give the simplest and known example of horizontal $W$-algebra.

Such example is provided by algebra $\mathcal{W}(2, k)$, which for integer $k$ is the symmetry algebra of CFT $\mathcal{M}(k, 2)$ with central charge $c_{k, 2}=\mathrm{c}_{2, k}=\frac{2(k-1)}{k+2}$. These models possess the symmetry generated by $s u(2)$ or $Z_{k}$ parafermions $\Psi_{i}(z), \Psi_{i}^{*}=\Psi_{k-i}$ [44] with spin $\frac{i(k-i)}{k}$. All parafermions and all symmetry algebra can be derived from $\Psi_{1}$ which has a representation by two fields $\varphi, \vartheta_{0}[43]$ :

$$
\Psi_{1}=\left(\sqrt{\frac{k+2}{k}} \mathrm{i} \partial \varphi+\partial \vartheta_{0}\right) e^{\sqrt{\frac{2}{k}} \vartheta_{0}}, \quad \Psi_{1}^{*}=\left(\sqrt{\frac{k+2}{k}} \mathrm{i} \partial \varphi-\partial \vartheta_{0}\right) e^{-\sqrt{\frac{2}{k}} \vartheta_{0}}
$$

The fields $\Psi_{1}(z), \Psi_{1}^{*}(z)$ commute with screening charges generated by $\left\{V_{1}, V_{-2}\right\}$, which for $b^{2}=-(n+k)$ are

$$
V_{1}=\exp \left(\mathrm{i} \sqrt{\frac{k+2}{2}} \varphi+\sqrt{\frac{k}{2}} \vartheta_{0}\right), \quad V_{-2}=\exp \left(\mathrm{i} \sqrt{\frac{k+2}{2}} \varphi-\sqrt{\frac{k}{2}} \vartheta_{0}\right)
$$

Hence all fields appearing in $\mathrm{OPE} \Psi_{1}(z) \Psi_{1}^{*}\left(z^{\prime}\right)$ commute with screenings and form $\mathcal{W}(2, k)$. Explicitly we have:

$$
\begin{aligned}
\left(z^{\prime}-z\right)^{2 \Delta_{1}} \Psi_{1}\left(z^{\prime}\right) \Psi_{1}^{*}(z) & =I+\left(z^{\prime}-z\right)^{2} \frac{k+2}{k} T(z) \\
& +\left(z^{\prime}-z\right)^{3}\left(\mathcal{W}_{3}(z)+\frac{T^{\prime}(z)}{2}+\right)+\cdots
\end{aligned}
$$

where $T(z)=-\frac{1}{2}\left((\partial \varphi)^{2}+(\partial \phi)^{2}\right)+\frac{\mathrm{i}}{\sqrt{2(k+2)}} \partial^{2} \varphi$ and the local current $\mathcal{W}_{3}(z)$ generating $\mathcal{W}(2, k)$ algebra up to numerical factor is

$$
\begin{aligned}
\mathcal{W}_{3}(z) & =\partial \phi\left(\sqrt{\frac{2}{9}}(3 k+4)(\partial \phi)^{2}+\sqrt{2}(k+2)(\partial \varphi)^{2}+\mathrm{i}(2+k)^{3 / 2} \partial^{2} \varphi\right) \\
& +\mathrm{i} \partial^{2} \phi \partial \varphi\left(k(2+k)^{1 / 2}\right)+\partial^{3} \phi \frac{1}{3 \sqrt{2}}
\end{aligned}
$$


More complicated field $\mathcal{W}_{3}(z)$ generating $\mathcal{W}(3, k)$ is given in appendix $\mathrm{A}$.

2. The CFTs $\mathcal{M}(k, n)$ and $\mathcal{M}(n, k)$ describe the critical points of RG flows. It is interesting to consider the integrable and non-integrable QFTs corresponding to perturbed $\mathcal{M}(k, n)$ $(\mathcal{M}(n, k)) \mathrm{CFTs}$, in particular, the perturbation considered at the end of section 8 (the tachyon $T)$ with $\gamma$ given by (90). In this case we derive the integrable QFTs corresponding to perturbation operator $\Phi_{k}(a d, 0)(\mathbf{\Phi}(\mathbf{a d}, \mathbf{0}, 0))$ with dimension $\Delta_{a d}=\frac{n}{k+n}$. The scattering theory for these QFTs was constructed in [37.

More interesting are the perturbations corresponding to deformed $C P(n-1)$-sigma models and the same models with fermion. First we consider the model which is non-integrable for $n>2$, but for $k \gg n$ has all characteristic properties of $C P(n-1)$-sigma model without fermion. We introduce the perturbing operators $\Lambda_{f}$ and $\Lambda_{f}^{*}$, where fields $\Lambda_{f}=\Phi_{k}\left(0, \omega_{1}\right)$ and $\Lambda_{f}^{*}=\Phi_{k}\left(0, \omega_{k-1}\right)$, associated with the vector representations of $s u(k)$, have left and right dimensions $\Delta_{f}=1-\frac{(k+1) n}{2 k(k+n-1)}$ and the theory with action

$$
\mathcal{A}_{\theta}=\mathcal{A}_{C F T}+\lambda \int\left(e^{\mathrm{i} \theta \pi / k} \Lambda_{f}+e^{-\mathrm{i} \theta \pi / k} \Lambda_{f}^{*}\right) d^{2} x
$$

For $n=2, \Lambda_{f}=\Psi_{1} \bar{\Psi}_{1}, \Lambda_{f}^{*}=\Psi_{1}^{*} \bar{\Psi}_{1}^{*}$ and the theory is integrable [31], 335] for $\theta=0$ and $\theta=\pi$. In the limit $k \rightarrow \infty$, it coincides with $O(3)$ or $C P(1)$-sigma model with parameter $\theta$, coupled with topological charge as $\mathrm{i} \theta T$. It was shown that for $\theta=0$ the theory is massive and for $\theta=$ $\pi$ massless. The effective central charge of the theory living on the finite space circle of length $R$, flows from 2 (in UV) to 0 (in IR) for $\theta=0$ and from 2 to 1 for $\theta=\pi$. The crucial role in this behavior is played by the instanton contributions. Similar contributions appear for $n>2$. The effective central charge $c_{\text {eff }}(R)$ has two types of contributions. The perturbative contributions and "instanton" ones. The first come in the perturbed CFT from the correlation functions

$$
\lambda^{2 m}\left\langle\Lambda_{f}\left(x_{1}\right) \Lambda_{f}^{*}\left(x_{2}\right), \ldots, \Lambda_{f}\left(x_{2 m-1}\right) \Lambda_{f}^{*}\left(x_{2 m}\right)\right\rangle
$$

and the second from the correlation functions

$$
e^{\mathrm{i} \theta \pi q} \lambda^{2 m+k q}\left\langle\Lambda_{f}\left(y_{1}\right) \Lambda_{f}\left(y_{2}\right) . . \Lambda_{f}\left(y_{k q}\right) ; \Lambda_{f}\left(x_{1}\right) \Lambda_{f}^{*}\left(x_{2}\right), . ., \Lambda_{f}\left(x_{2 m-1}\right) \Lambda_{f}^{*}\left(x_{2 m}\right)\right\rangle
$$

These correlation functions do not vanish because in CFTs $\mathcal{M}(k, n)$ the fields $\Lambda_{f}$ and $\Lambda_{f}^{*}$ have $Z_{k}$ charge 1 and -1 . As a result the effective central charge can be expanded as

$$
c_{\mathrm{eff}}^{(\theta)}(R)=c_{n, k}+\sum_{q=0, m=0}^{\infty} \cos (\pi \theta q) d_{m}^{(q)}\left(M_{s} R\right)^{(2 k q+4 m)\left(1-\Delta_{f}\right)}
$$

where $1-\Delta_{f}=\frac{(1+k) n}{2 k(k+n-1)}=\frac{n}{2 k}(1+O(1 / k)) ; c_{n, k}=2(n-1)(1+O(1 / k))$ and mass parameter $M_{s} \sim \lambda^{\frac{1}{2\left(1-\Delta_{f}\right)}}$. All coefficients $d_{m}^{(q)}$ can be derived by integration of correlation functions (99100). These series are very similar to those for $n=2$. For example, the first instanton contribution (up to the factor $d_{0}^{(1)}$, which can be calculated exactly) is proportional $\left(M_{s} R\right)^{n}$, in agreement with renormalization group for $C P(n-1)$ QFT. It is natural to expect that for $\theta=0$ the QFT (98) will be massive and for $\theta=\pi$ it will be massless.

To derive the integrable QFT describing in the limit $k \rightarrow \infty, C P(n-1)$-model with fermion, we take into account the field $\vartheta_{n}$ which was completely irrelevant in the action $\mathcal{A}_{C F T}$ 
(6). The field $\vartheta_{n}(x)=\theta(x)$ will play a role of fluctuating topological parameter $\theta$. We introduce the free massless field $\theta(x)$ and modify the fields $\left(\Lambda_{f}(x), \Lambda_{f}^{*}(x)\right) \in \mathcal{M}(k, n)$ as

$$
\Lambda=\Lambda_{f} \exp \left(\mathrm{i} \nu_{n, k} \theta(x)\right), \quad \Lambda^{*}=\Lambda_{f}^{*} \exp \left(-\mathrm{i} \nu_{n, k} \theta(x)\right), \quad \nu_{n, k}^{2}=\left(\frac{(n+k)}{k(k+n-1)}\right)
$$

The fields $\left(\Lambda(x), \Lambda^{*}(x)\right)$ have the right and left dimensions $\Delta=1-\frac{n-1}{2(n+k-1)}$. The QFT with action

$$
\mathcal{A}_{\theta(x)}=\mathcal{A}_{C F T}+\int\left(\frac{\partial_{\mu} \theta(x) \partial_{\mu} \theta(x)}{8 \pi}+\varkappa\left(\Lambda+\Lambda^{*}\right)\right) d^{2} x
$$

is integrable. Its scattering theory can be derived by the quantum group restriction from the $S$-matrix described in section 4 . With this scattering theory we can write the TBA equations for effective central charge. The massless field $\theta(x)$ cancels all "instanton" contributions, and the series for $c_{\text {eff }}(R)$ simplify

$$
c_{\text {eff }}(R)=c_{n, k}+1+\sum_{m=2}^{\infty} \mathrm{d}_{m}(M R)^{2 \frac{m(n-1)}{k+n-1}} .
$$

The physical mass $M$ is related with coupling constant $\varkappa$ as follows

$$
\frac{\pi \varkappa \Gamma\left(\frac{1}{n+k-1}\right)}{\Gamma\left(1-\frac{1}{n+k-1}\right) k}=\left(\frac{n(n-1)}{2}\right)^{1 / 2}\left(M \frac{\Gamma\left(\frac{1}{n}\right) \Gamma\left(\frac{n+k-1}{n}\right)}{4 \Gamma\left(\frac{k}{n}\right)}\right)^{\frac{(n-1)}{(n+k-1)}}
$$

Using this relation and properties of the coefficients $\mathrm{d}_{m}$, it is possible to calculate the scaling limit $(k \gg 1, \log 1 / M R) \gg 1 \cdot \frac{\log (1 / M R)}{k}$ fixed) of the effective central charge

$$
c_{\mathrm{eff}}(R)=2 n-1+\frac{3 n(n-1)}{k}\left(1-(M R)^{\frac{2(n-1)}{(n+k-1)}}\right)^{-1} .
$$

In the limit $k \rightarrow \infty, c_{\text {eff }}(R)=1+2(n-1)-\frac{3 n}{2 \log (1 / M R)}$. This result coincides with RG equation for $C P(n-1)$ model with fermion.

We note that for constant $\theta(x)=\theta \frac{\pi}{k \nu}$ the QFTs (101) and (98) coincide. If we consider the action of $C P(n-1)$-model with fermion

$$
\mathcal{A}_{C P(n-1)+\chi}=\mathcal{A}_{C P(n-1)}+\frac{1}{4 \pi} \int d^{2} x\left(\mathrm{i} \bar{\chi} \gamma_{\mu}\left(\partial_{\mu}+B_{\mu}\right) \chi\right)
$$

and use the relations $i \bar{\chi} \gamma_{\mu} \partial_{\mu} \chi=\frac{1}{2} \partial_{\mu} \theta(x) \partial_{\mu} \theta(x)$ and $\bar{\chi} \gamma_{\mu} \chi=\varepsilon_{\mu \sigma} \partial_{\sigma} \theta(x)$ then after the integration by parts with identity $\varepsilon_{\mu \sigma} \partial_{\sigma} B_{\mu}=4 \pi T(x)$, where $T(x)$ is the density of topological charge we derive

$$
\mathcal{A}_{C P(n-1)+\chi}=\mathcal{A}_{C P(n-1)}+\int d^{2} x\left(\frac{\partial_{\mu} \theta(x) \partial_{\mu} \theta(x)}{8 \pi}+\mathrm{i} \theta(x) T(x)\right) .
$$

We see that field $\theta(x)$ plays the role of axion [26]. For constant $\theta(x)$ this action coincides with the action of $C P(n-1)$-model with topological term. It has very rich dynamics, but does not define the integrable QFT .

3. Studying the deformed SMs on the groups and on the symmetric spaces it is useful to consider the extended set of perturbed rational CFTs. Here we consider the well known 
rational coset CFTs $\mathcal{M}(G, m, l)=\frac{G_{m} G_{l}}{G(m+l)}$, where $G$ is the simply laced Lie algebra, perturbed by integrable field $\Phi$ associated with the adjoint representation of $G$ and conformal dimension $\Delta=1-\frac{h}{m+l+h}$. The central charge of $\mathcal{M}(G, m, l)$ is

$$
c(G, m, l)=\frac{r(h+1) l m(l+m+2 h)}{(l+m+h)(m+h)(l+h)}
$$

where $r$ and $h$ are rank and Coxeter number of $G, D=r(h+1)=\operatorname{dim}(G)$.

The action of integrable perturbed $\mathrm{CFT} \mathcal{M}(G, m, l)$ is

$$
\mathcal{A}_{G}=\mathcal{A}_{G}(C F T)-\kappa \int \Phi(x) d^{2} x
$$

For $\kappa>0$ these QFTs are massive, the particles for finite $m, l$ are the kinks, and exact relations between the masses of these particles and coupling constant $\kappa$ are known [38] .

Here we consider the relations between QFTs (105) and SMs in different regions of integers $m, l$ and $h$. The simplest region corresponds to $m>>l$. In the limit $m \rightarrow \infty$ our QFT can be written as

$$
\mathcal{A}_{G}=\mathcal{A}_{G}\left(W Z W_{l}\right)-\kappa_{1} \int \sum J_{a}(z) \overline{J_{a}}(\bar{z}) d^{2} x
$$

i.e. Wess-Zumino-Witten model at level $l$ perturbed by currents. For finite $m>>l$, QFT (105) describes the deformation of this SM. The scaling function for the effective central charge on a circle of length $R$ was calculated in [38] and is given by

$$
\begin{aligned}
c_{\mathrm{eff}}(R)-c(G, m, l) & =-2 D h \frac{l^{2} m^{3}}{h^{3}}(M R)^{4 h /(m+l+h)}+\cdots \quad m<<\log (1 / M R) \\
& =\frac{1}{2} D l^{2} / \log ^{3}(1 / M R)+\cdots \quad m>>\log (1 / M R)
\end{aligned}
$$

in agreement with conformal perturbation theory and RG.

Another region depends on two parameters $m>>h, l>>h$. It is similar to the twoparameter deformations $\left(p_{1}, p_{2}\right)$ of principal chiral field (section 6). The QFT (105) in this region describes the integrable deformations of the principal chiral field considered as perturbed CFT. It is convenient to introduce parameters $\mathrm{k}=\frac{l-m}{l+m+2 h}$ and $\mathrm{y}=\frac{h}{l+m+2 h}<<1$. The scaling function for the effective central charge in this region was calculated for $G=S U(2)$ and conjectured for all $G$ in [16]. The result can be expressed through function $\tau(R)$ where

$$
\left(\frac{1-\tau}{1+\tau}\right)\left(\frac{1+\mathrm{k} \tau}{1-\mathrm{k} \tau}\right)^{\mathrm{k}}=(M R)^{2 \mathrm{y}}
$$

Namely,

$$
c_{\mathrm{eff}}(R)-c(G, m, l)=-\frac{D \mathrm{y}}{\left(1-\mathrm{k}^{2}\right) 2 \tau}\left(\left(3+\mathrm{k}^{2}\right)(1-\tau)^{2}-\mathrm{k}^{2}\left(1-\tau^{2}\right)^{2}\right) .
$$

This function has the asymptotics

$$
-D y\left(6\left(\frac{1-\mathrm{k}}{1+\mathrm{k}}\right)^{2 \mathrm{k}}(M R)^{4 \mathrm{y}}+\frac{64}{\left(1-\mathrm{k}^{2}\right)}\left(\frac{1-\mathrm{k}}{1+\mathrm{k}}\right)^{3 \mathrm{k}}(M R)^{6 \mathrm{y}}+\cdots\right)
$$

\footnotetext{
${ }^{6}$ The scattering matrix of the basic kinks is derived from that for deformed principle chiral field (see footnote 3) equal to $S(G)_{p_{1}, p_{2}}(\theta)$, where $p_{1}=h+m, p_{2}=h+l$, by the quantum group restriction with respect to symmetry $G_{\mathrm{q}_{1}} \otimes G_{\mathrm{q}_{2}}$.
} 
for $y \log \left(\frac{1}{M R}\right)>>1$. The first two terms in this expansion were calculated exactly in [38]. The result coincides with (108) up to y $\log \mathrm{y}$.

In the limit $y \log \left(\frac{1}{M R}\right)<<1$, corresponding to the principal chiral field we get

$$
c_{\text {eff }}(R)-D=-\frac{3 D}{2 \log \left(\frac{1}{M R}\right)}+O\left(y \log \left(\frac{1}{M R}\right)\right)
$$

in agreement with RG for principal chiral field.7

It is interesting also to consider the perturbed $\mathcal{M}(G, m, l)$ CFTs in the region $h>>1$, $m$, $l$, fixed. We consider here $G=S U(k), O(k)(h=k, k-2)$. The central charge (104) will be now $2 m l+O\left(\frac{1}{h}\right)$ and $m l+O\left(\frac{1}{h}\right)$ for $S U(k)$ and $O(k)$. The perturbation field $\Phi_{f}$ now will be associated with the fundamental (vector) representation $f$ of $G$.

For $G=S U(k)$ and $m=1, \Phi_{f}=\Lambda_{f}$ and in general case we use as the perturbation field $\Phi(\theta)=e^{\mathrm{i} \theta / k} \Phi_{f}+e^{-\mathrm{i} \theta / k} \Phi_{f}^{*}$ with dimension $1-\Delta_{f}=\frac{1+k m}{2(k+m)}+\frac{1+k l}{2(k+l)}=\frac{m+l}{k}+O\left(\frac{1}{k^{2}}\right)$. Here $\theta$ is a topological parameter. This QFT will have the "instanton" and perturbative contributions and in the limit $k \rightarrow \infty$ it will coincide with $\frac{U(l+m)}{U(l) U(m)}$ SM with the topological parameter. This QFT is Kähler SM and possesses the instantons. It is natural to assume that it will be massive for $\theta=0$ and massless for $\theta=\pi$ and if we make topological parameter $\theta$ to be fluctuating field $\theta(x)$ (axion), the corresponding QFT will be integrable.

For $G=O(k)$ and $m=1$ the CFT perturbed by $\Phi_{f}$ was studied by Fendley [40]. For general $m$ field $\Phi_{f}$ has the dimension $1-\Delta_{f}=\frac{1}{2}\left(\frac{m-1}{m+k-2}+\frac{l-1}{l+k-2}\right)$. In the limit $k \rightarrow \infty$ this QFT describes integrable $\frac{O(l+m)}{O(l) O(m)}$ sigma models.

\section{Concluding Remarks}

In this section we provide some notes which can useful for studying dual representations of deformed SMs on symmetric spaces.

1. In this paper we did not considered the massless RG flows which have the same UV asymptotics as the deformed principle chiral SMs and IR asymptotics corresponding to rational CFTs. These Ricci flows are related with SMs with singular metrics and will be studied in a separate publication. In the previous section we have shown that integrable perturbed rational coset models $\mathcal{M}(G, m, l)(105)$ with $m, l \gg h$ and $\kappa>0$ describe the massive deformed principle chiral SMs. The massless RG flows in perturbed coset models $\mathcal{M}(G, m, l)$ with $m, l \gg h, m \gg l$ are described by the action (105) with $\kappa<0$. The effective central charge $c_{\text {eff }}(R)$ for these flows is defined by eq. (107) where one should make the substitution $\tau \rightarrow \tau_{-}$, where $\tau_{-}(R)$ is defined by the relation

$$
\left(\frac{1-\tau_{-}}{1+\tau_{-}}\right)\left(\frac{1+\mathrm{k} \tau_{-}}{1-\mathrm{k} \tau_{-}}\right)^{\mathrm{k}}=-(M R)^{2 \mathrm{y}}
$$

It has the following UV and IR asymptotics:

$$
c_{\text {eff }}(R)-c(G, m, l)=-D \mathrm{y}\left(6\left(\frac{1-\mathrm{k}}{1+\mathrm{k}}\right)^{2 \mathrm{k}}(M R)^{4 \mathrm{y}}-\frac{64}{\left(1-\mathrm{k}^{2}\right)}\left(\frac{1-\mathrm{k}}{1+\mathrm{k}}\right)^{3 \mathrm{k}}(M R)^{6 \mathrm{y}}+\cdots\right)
$$

${ }^{7}$ The UV asymptotics (109) in the region $y \log \left(\frac{1}{M R}\right)<<1$ (small deformations) is universal and for perturbed CFT (105) and SM (52) it is the same. On the other hand, for these theories the UV asymptotics in the region $\mathrm{y} \log \left(\frac{1}{M R}\right)>>1$ are different (see [17, [16] and compare eqs. (81) and (102)). This difference is related with the fact that the quantum group restriction cancels the contribution of zero modes, which provide the main term in the asymptotics of non-restricted SMs. 
for $(M R) \ll 1$ and

$$
c_{\mathrm{eff}}(R)-c(G, m-l, l)=\frac{D \mathrm{y}}{\mathrm{k}}\left(6\left(\frac{1-\mathrm{k}}{1+\mathrm{k}}\right)^{\frac{2}{\mathrm{k}}}(M R)^{\frac{4 \mathrm{y}}{\mathrm{k}}}-\frac{64}{\left(1-\mathrm{k}^{2}\right)}\left(\frac{1-\mathrm{k}}{1+\mathrm{k}}\right)^{\frac{3}{\mathrm{k}}}(M R)^{\frac{6 \mathrm{y}}{\mathrm{k}}}+\cdots\right)
$$

for $(M R) \gg 1$, i.e. these $\mathrm{RG}$ flows relate $\mathrm{CFTs} \mathcal{M}(G, m, l)$ and $\mathcal{M}(G, m-l, l)$.

2. In the Introduction we noted that SMs with nice duality form a very small subspace in the space of integrable SMs on the deformed symmetric spaces. Here we give a simple example of the integrable QFT, which possesses a duality, but not nice duality. This example is provided by $N=2$ supersymmetric Sine-Gordon model. Its QFT representation fits the twoparameter family of integrable theories (18) where one of the parameters is fixed as $a_{1}^{2}=2$. Similarly, its SM representation (45) is described by the two-parameter family with $p_{2}=2$. This theory tends to $N=2$ supersymmetric $O(3)$ (or $C P(1)$ ) in the limit $a_{1} \rightarrow \infty$, but it does not have the weak coupling region. However, as it was noted in section 6 , even in this case the QFT representation (18) is useful because it helps to reconstruct the $S$-matrix. As can be seen from equations given in (19), the currents $J_{2}^{( \pm)}$have the spin $\frac{3}{2}$ and generate $N=2$ supersymmetry. The currents $J_{1}^{( \pm)}$generate $S U(2)_{\mathrm{q}}$ symmetry with q $=\exp \left(\mathrm{i} \frac{2 \pi}{a_{1}^{2}}\right)=\exp \left(\mathrm{i} \frac{2 \pi}{p_{1}}\right)$ that fixes $S$-matrix (20).

3. All known "nice" dual theories do not have the UV divergences in the perturbation theory. In particular, the bulk vacuum energy is UV finite (see (38)). This implies the following equality

$$
\sum_{i=1}^{n_{b}} M_{b, i}^{2}=\sum_{i=1}^{n_{f}} M_{f, i}^{2}
$$

where $M_{b, i}^{2}$ and $M_{f, i}^{2}$ are the masses of bosonic and Majorana fermionic particles in the weak coupling region $b \ll 1$. This condition ensures the vanishing of the one-loop UV divergence in the bulk vacuum energy

$$
\frac{1}{2} \sum_{i=1}^{n_{f}} M_{f, i}^{2} \log L-\frac{1}{2} \sum_{i=1}^{n_{b}} M_{b, i}^{2} \log L
$$

The theory is integrable. This implies the existence of an integrable purely bosonic QFT which is obtained by setting all fermions to zero in the weak coupling region. The simplest examples of such QFT are provided by affine Toda theories, for which the 1.h.s. of (110) is known. In the strong coupling (SM) region we expect that some bosonic particles disappear from the spectrum, whereas the stable bosonic particles and Majorana fermions have the same mass $M$ and form the multiplet of the group in the numerator of the coset. The dimension of such multiplet equals $D_{m}=n_{f}+n_{b}^{(s)}$, where $n_{b}^{(s)}$ is the number of stable bosons. The UV central charge of such QFT is given by $c_{U V}=n_{b}+\frac{1}{2} n_{f}$. These equalities together with (110) put rather rigid restrictions on the "nice" dual theories with affine Toda bosonic part in the weak coupling region 8 It is easy to see that for the theories considered in this paper and in [22] they are satisfied.

${ }^{8}$ If we assume that the unstable particles in the weak coupling region are the bound states of fermionic particles and stable particles have the same mass $M$ as all fermionic particles (see [22]) we derive for affine Toda QFTs with the affine Lie algebras $\mathfrak{g}_{n}$ of rank (or number of bosonic particles) $n=n_{b}$ the following results. Namely, $\mathfrak{b}_{n}: \sum m_{b}^{2}=(2 n-1) M^{2}, n_{b}^{(s)}=2 ; \mathfrak{d}_{n}: \sum m_{b}^{2}=(2 n-2) M^{2}, n_{b}^{(s)}=2 ; \mathfrak{c}_{n, \text { dual }}: \sum m_{b}^{2}=M^{2}, n_{b}^{(s)}=0$. The cases $\mathfrak{g}_{n}=\mathfrak{b}_{n, \text { dual }}, \mathfrak{c}_{n}, \mathfrak{a}_{n}$ were considered in [22] and in this paper. 
4. Here we consider conditions for the existence of an infinite family of integrable QFTs with "nice" duality without assumptions about purely bosonic part and UV convergence in perturbation theory. It means that such QFTs can be described by weak coupling theories in one regime and by slightly deformed SMs on the symmetric spaces in the dual regime. Of course, when we know scattering theory of these QFTs for all values of coupling parameters, the necessary condition can be easily formulated. There should be a point in the space of parameters where the scattering matrix is the identity. If we consider the asymptotically free SMs on the coset spaces with one group $G$ in the numerator, the scattering matrix should have the symmetry of this group $G$ (or $G \times G$ in the case of the principle chiral field) and the particles with minimal mass should belong to some representation $R_{\min }$ of Yangian of $G$ (or $G \times G$ for principal chiral field) with dimension $D_{\text {min }}$. Let $N_{\text {min }}=D_{\text {min }}$ for real $R_{\text {min }}$ and $N_{\text {min }}=2 D_{\text {min }}$ for complex $R_{\min }$. For QFTs with "nice" duality the particles in the weak coupling region are described by a set of almost free fields in the action. A part of these particles disappears from the spectrum during the evolution from weak to strong coupled QFT. In any case, even if we assume that all particles survive in the strong coupling regime and were described by fermionic fields in the weak coupling regime, we get a rather rough inequality

$$
c_{U V} \geq \frac{1}{2} N_{\min }
$$

This inequality excludes all principle chiral field QFTs, besides $G=S U(2)$ and all reasonable

coset SMs besides $\frac{O(n)}{O(n-1)}$ considered in [22], $\frac{S U(n)}{S U(n-1)}$ considered in this paper and $\frac{S p(n)}{S p(n-1)}$ which will be considered elsewhere.

\section{Acknowledgements}

The author is very grateful to Alexey Litvinov for useful discussions and Sergei Alexandrov for kind help in preparation of this manuscript for publication.

\section{A Explicit expressions for the currents and densities of local integrals}

In this Appendix we provide the expressions for the current $\mathcal{W}_{3}$ for CFT (6) with $n=3$ and the local integrals of motion for QFTs (15) with $n=3$. We choose the two dimensional vectors $h_{i}$ (the weights of fundamental representation of $s u(3)$ ), and one dimensional $\eta_{i}$ (the weights of the fundamental representation of $s u(2))$ in the form:

$$
h_{1}=\left(\frac{1}{\sqrt{6}}, \frac{1}{\sqrt{2}}\right), \quad h_{2}=\left(\frac{-2}{\sqrt{6}}, 0\right), \quad h_{3}=\left(\frac{1}{\sqrt{6}}, \frac{-1}{\sqrt{2}}\right), \quad \eta_{1}=-\eta_{2}=\frac{1}{\sqrt{2}}
$$

Then the current $\mathcal{W}_{3}(3, b)$ has the form

$$
\begin{aligned}
\mathcal{W}_{3} & =\sqrt{6} b \partial \varphi_{1}\left(b c\left(\partial \varphi_{1}\right)^{2}+\mathrm{i} \sqrt{6} b^{2} \partial \varphi_{1} \partial \vartheta_{0}-3 c b\left(\partial \varphi_{2}\right)^{2}+\sqrt{2} c a\left(a \partial^{2} \varphi_{2}+2 \mathrm{i} b \partial^{2} \vartheta_{3}\right)\right) \\
& +3 \mathrm{i} b \partial \vartheta_{0}\left(2 b^{2}\left(\partial \varphi_{2}\right)^{2}+a\left(3 a\left(\partial \vartheta_{3}\right)^{2}+\left(3+5 b^{2}\right)\left(\partial \vartheta_{0}\right)^{2}-\sqrt{2} b\left(2 b \mathrm{i} \partial^{2} \vartheta_{3}+a \partial_{2}^{2} \partial^{2} \varphi_{2}\right)\right)\right. \\
& +9 \sqrt{2} a b \partial^{2} \vartheta_{0}\left(b \partial \vartheta_{3}-\mathrm{i} a \partial \varphi_{2}\right)-3 \sqrt{6} c \partial^{3} \varphi_{1},
\end{aligned}
$$


where $a=\sqrt{1+b^{2}}, c=\frac{1}{\sqrt{6}} \sqrt{3+b^{2}}$. The current $\mathcal{W}_{3}(3, k)$ can be derived from (111) by substitution $b^{2}=-3-k$.

The local integral of motion for QFT (15) with $n=3$ is

$$
\begin{aligned}
\mathcal{I}_{3}^{(3)} & =\partial \varphi_{1}\left(\sqrt{2} b\left(\left(\partial \varphi_{1}\right)^{2}-3\left(\partial \varphi_{2}\right)^{2}\right)-6\left(a^{2} \partial^{2} \varphi_{2}+\mathrm{i} a b\left(\sqrt{3} \partial^{2} \phi_{1}+\partial^{2} \phi_{2}\right)\right)\right. \\
& +2 \mathrm{i} \partial \phi_{0}\left(3 b^{2}\left(\left(\partial \varphi_{1}\right)^{2}+\left(\partial \varphi_{2}\right)^{2}\right)+3 a^{2}\left(\left(\partial \phi_{1}\right)^{2}+\left(\partial \phi_{2}\right)^{2}\right)+\left(b^{2}+a^{2}\right)\left(\partial \phi_{0}\right)^{2}\right) \\
& -\mathrm{i} \sqrt{2} a \partial \phi_{1}\left(\left(\partial \phi_{1}\right)^{2}-3\left(\partial \phi_{2}\right)^{2}\right)-6 \mathrm{i} a b \partial \varphi_{2}\left(\partial^{2} \phi_{1}-\sqrt{3} \partial^{2} \phi_{2}\right)-6 a^{2} \partial \phi_{1} \partial^{2} \phi_{2}
\end{aligned}
$$

here $a=\sqrt{1+b^{2}}$.

\section{B Possible parametrization of $\mathrm{SU}(n)$}

In this Appendix we describe the possible parametrization of matrix $g \subset S U(n)$. The generators of $S U(n)$ can be numerated as $T_{i j}^{(+)}, T_{i j}^{(-)}$with $n \leq j>i \geq 1$ and $H_{i}, i=1, . ., n-1$ where $H_{i}$ are the real diagonal matrices $\operatorname{tr}\left(H_{i} H_{j}\right)=2 \delta_{i j}$. Let $E_{i, j}(k, m)=\delta_{i k} \delta_{j m}$ then

$$
T_{i j}^{(+)}=E_{i j}+E_{j i}, \quad T_{i j}^{(-)}=\mathrm{i}\left(E_{i j}-E_{j i}\right)
$$

The matrix $g \subset S U(n)$ can be written as

$$
g=\prod_{k=1}^{n-1} e^{\mathrm{i} \eta_{k} H_{k}} \prod_{j>i}^{n} e^{\mathrm{i}\left(a_{i j} T_{i j}^{(+)}+b_{i j} T_{i j}^{(-)}\right)}
$$

The multipliers in this product can be easily calculated, for example, for $n=2, T_{i j}^{(+)}=$ $\tau_{1}, T_{i j}^{(-)}=\tau_{2}$ and

$$
g=e^{\mathrm{i} \eta_{1} \tau_{3}}\left(\begin{array}{cc}
\cos \theta_{12} & \mathrm{i} e^{-\mathrm{i} \beta_{12}} \sin \theta_{12} \\
-\mathrm{i} e^{\mathrm{i} \beta_{12}} \sin \theta & \cos \theta_{12}
\end{array}\right)=e^{\mathrm{i} \eta_{1} \tau_{3}} \mathrm{~g}_{12}\left(\theta_{12}, \beta_{12}\right)
$$

where $\theta_{12}=\sqrt{a_{12}^{2}+b_{12}^{2}}$ and $e^{\mathrm{i} \beta_{12}}=\frac{a_{12}+\mathrm{i} b_{12}}{a_{12}-\mathrm{i} b_{12}}$. It is easy to generalize this expression to any multiplier $e^{\mathrm{i}\left(a_{i j} T_{i j}^{(+)}+b_{i j} T_{i j}^{(-)}\right)}$. We denote $\mathrm{g}_{i j}\left(\theta_{i j}, \beta_{i j}\right)=e^{\mathrm{i}\left(a_{i j} T_{i j}^{(+)}+b_{i j} T_{i j}^{(-)}\right)}$then $g \subset S U(n)$ can be written as

$$
g=\prod_{k=1}^{n-1} e^{\mathrm{i} \eta_{k} H_{k}} \prod_{j>i}^{n} \mathrm{~g}_{i j}\left(\theta_{i j}, \beta_{i j}\right)
$$

We note that order of multipliers in this product can be taken arbitrary. It leads to different parametrizations of $g$.

\section{References}

[1] Coleman S., The Quantum Sine-Gordon Equation as the Massive Thirring Model, Phys.Rev. D11 (1975) 2088-2097

[2] Mandelstam S., Soliton Operators for the Quantized Sine-Gordon Equation, Phys.Rev. D11 (1975) 3026-3030 
[3] Zamolodchikov A., Comm. Math. Phys. 55 (1977) 183-195

[4] Arinstein A., Fateev V. and Zamolodchikov A, Quantum S Matrix of the (1+1)Dimensional Toda Chain, Phys. Lett. B 89 (1979) 389-395

[5] Braden H., Corrigan E., Dorey P., Sasaki R., Affine Toda Field Theory and Exact S Matrices, Nucl .Phys.B 338 (1990) 689-746

[6] Delius G., Grisaru M., Zanon N., Exact S matrices for nonsimply laced affine Toda theories, Nucl. Phys. B 382 (1992) 365-406, hep/th 9201067

[7] Montonen C., Olive D., Magnetic Monopoles as Gauge Particles, Phys. Lett. B 125 (1977) $117-124$

[8] Goddard P., Nuyts J., Olive D., Gauge Theories and Magnetic charge, Nucl. Phys. B 125 (1977) $1-28$

[9] Seiberg N., Witten E., Electric - magnetic duality, monopole condensation, and confinement in N=2 supersymmetric Yang-Mills theory,Nucl. Phys. B 426 (1994) 19-52, hep/th 9711200

[10] Maldacena J., The Large N limit of superconformal field theories and supergravity, Int. J. Theor. Phys. 38, 1113 (1999) 1113-1133

[11] Maldacena J., The Large N limit of superconformal field theories and supergravity, Adv. Theor. Math. Phys. 2, (1998) 231 -252, hep/th

[12] Freedan D., Nonlinear Models in $2+\varepsilon$ dimension, Phys. Rev. Lett. 45 (1980) 1057-1060

[13] Fateev V., Frolov I., Shwarz A., Quantum Fluctuations of Instantons in Two-dimensional Nonlinear Theories, Sov.J.Nucl.Phys. 30 (1979) 590, Yad.Fiz. 30 (1979) 1134-1147

[14] Irving A., Michael C., The spectrum and potential in CP(N), Nucl. Phys. B 371 (1992) $521-536$

[15] D'Adda A., Luscher M., Di Vecchia P., A 1/n Expandable Series of Nonlinear Sigma Models with Instantons, Nucl.Phys. B146 (1978) 63-76

[16] Fateev V., The sigma model (dual) representation for a two-parameter family of integrable quantum field theories, Nucl.Phys. B473 (1996) 509-538

[17] Fateev V., Onofri E., Zamolodchikov Al., The Sausage model (integrable deformations of O(3) sigma model), Nucl.Phys. B406 (1993) 521-565

[18] Fateev V., The duality between two-dimensional integrable field theories and sigma models, Phys. Lett. B 357 (1995) 397-403

[19] Klimčik C., On integrability of the Yang-Baxter sigma-model, J.Math.Phys. 50 (2009) 043508 arXiv:0802.3518 [hep-th]

[20] Delduc F., Malgro M., Vicedo B., On classical q-deformations of integrable sigma-models, JHEP 1311 (2013) 192 arXiv:1308.3581 [hep-th] 
[21] Altschuler D., Quantum Equivalence of Coset Space Models, Nucl.Phys. B313 (1989) 293

[22] Fateev V., Litvinov Al., Integrability, Duality and Sigma Models, arXiv:1804.03399 [hepth]

[23] Fateev V., Integrable Deformations of Sine-Liouville Conformal Field Theory and Duality, SIGMA 13 (2017) 080 arXiv:1705.06424 [hep-th]

[24] Hoare B., Tseytlin A., On integrable deformations of superstring sigma models related to AdSn×Sn supercosets, Nucl.Phys. B897 (2015) 448-478 arXiv:1504.07213 [hep-th]

[25] Litvinov A., Integrable $\operatorname{gl}(\mathrm{n} \mid \mathrm{n})$ Toda field theory and its sigma-model dual, arXiv:1901.04799

[26] Balog J., Forgacs P., Palla L., A Two-dimensional integrable axionic sigma model and T duality,Phys. Lett. B 484 (2000) 367 hep-th/0004180

[27] Baseilhac P, Fateev V., Expectation values of local fields for a two-parameter family of integrable models and related perturbed conformal field theories, Nucl.Phys. B532 (1998) 567-587 hep-th/9906010

[28] Fateev V., Litvinov Al., Multipoint correlation functions in Liouville field theory and minimal Liouville gravity, Theor.Math.Phys. 154 (2008) 454-472 arXiv:07071664

[29] Zamolodchikov A., "Irreversibility" of the Flux of the Renormalization Group in a 2D Field Theory, JETP Lett. 43 (1986) 730-732.

[30] Ganin M., P., 1963. On a fredholm integral equation whose kernel depends on the difference of the arguments, Izv.Vuz.Mat., 2 (1963) 31-43

[31] Fateev V., Integrable deformations in $\mathrm{Z}(\mathrm{N})$ symmetrical models of conformal quantum field theory, Int.J.Mod.Phys. A6 (1991) 2109-2132

[32] Cherednik I., Relativistically Invariant Quasiclassical Limits of Integrable Twodimensional Quantum Models, Teor.Mat.Fiz. 47 (1981) 225-229

[33] Abdalla E., Integrable nonlinear sigma models with fermion, Commun. Math. Phys. 104, (1986) 123-138

[34] Abdalla E., Forger M., Gomes M., On the Origin of Anomalies in the Quantum Nonlocal Charge for the Generalized Nonlinear $\sigma$-Models, Nucl.Phys. B210 (1982) 181-192

[35] Fateev V., Zamolodchikov Al., Integrable perturbations of Z(N) parafermion models and O(3) sigma model, Phys.Lett. B271 (1991) 91-100

[36] Lund R., Regge T., Unified approach to string and vortices with soliton solutions, Phys. Rev. D 14 (1976) 1524-1535

[37] de Vega H., Fateev V., Factorizable S matrices for perturbed W invariant theories, Int.J.Mod.Phys. A6 (1991) 3221-3234 
[38] Fateev V., The Exact relations between the coupling constants and the masses of particles for the integrable perturbed conformal field theories, Phys.Lett. B324 (1994) 45-51

[39] Zamolodchikov Al., Mass scale in sine-Gordon model and it's reduction J.Mod.Phys. A10 (1995) 1125-1150

[40] Fendley P, Sigma models as perturbed conformal field theories, Phys.Rev.Lett. 83 (1999) 4468-4471 hep-th/9906036

[41] Fateev V., Normalization factors, reflection amplitudes and integrable systems, hep-th/0103014

[42] Zamolodchikov A., Zamolodchikov Al., Structure constants and conformal bootstrap in Liouville field theory, Nucl.Phys. B477 (1996) 577-605 hep-th/9506136

[43] Nemeshansky D., Feigin-Fuchs Representation of $S U(2)_{k}$ Kac-Moody Algebra, Phys.Lett. B224 (1989) 121-124

[44] Fateev V., Zamolodchikov A., Parafermionic Currents in the Two-Dimensional Conformal Quantum Field Theory and Selfdual Critical Points in Z(n) Invariant Statistical Systems, Sov.Phys.JETP 62 (1985) 215-225, Zh.Eksp.Teor.Fiz. 89 (1985) 380-399

[45] Changrim A, Baseilhac P., Fateev V., Kim C., Chaiho C. Reflection amplitudes in NonSimply Laced Toda theories and thermodynamic Bethe ansatz, Phys.Lett. B481 (2000) 114-124 hep-th/000213

[46] Fateev V., Onofri O., An Eigenvalue problem related to the nonlinear sigma model: Analytical and numerical results, J.Phys. A36 (2003) 11881-11900 math-ph/0307010

[47] Olshanetsky M.,Perelomov A., Classical integrable systems related to Lie algebras, Phys. Rep. 71 (1981) 313

[48] Basso B., Rej A., On the integrability of two-dimensional models with U(1)xSU(N) symmetry, Nucl.Phys. B866 (2013) 337-377 arXiv:1207.0413 [hep-th] 